\title{
On the Diversity of Noncoherent Distributed Space-Frequency Coded Relay Systems With Relay Censoring
}

\author{
Sung-En Chiu, Feng-Tsun Chien, Member, IEEE, and Ronald Y. Chang, Member, IEEE
}

\begin{abstract}
This paper considers a noncoherent distributed space-frequency coded (SFC) wireless relay system with multiple relays. Each relay adopts a censoring scheme to determine whether the relay will decode and forward the source's information toward the destination. We analytically obtain the achievable diversity for both cases of perfect and imperfect relay censoring. With perfect censoring, we show that the same diversity of a conventional noncoherent SFC MIMO-OFDM system is achievable in the considered noncoherent distributed SFC system with maximum-likelihood (ML) decoding, regardless of whether partial information of channel statistics and relay decoding status is available at the destination. With imperfect censoring, we analytically investigate how censoring errors affect the achievability of the system's diversity. We show that the two types of censoring errors, which correspond to useless and harmful relays, respectively, can decrease the achievable diversity significantly. Our analytical insights and numerical simulations demonstrate that the noncoherent distributed system can offer a comparable diversity as the conventional MIMO-OFDM system if relay censoring is carefully implemented.
\end{abstract}

Index Terms-Wireless relay networks, space-frequency codes (SFC), noncoherent communication, decode-and-forward (DF), cooperative diversity.

\section{INTRODUCTION}

C OOPERATIVE relaying where multiple intermediate relays cooperatively help forward the source's information toward the destination is a promising technique to enhance the coverage and reliability of wireless communication [1], [2]. In a cooperative relay network, different nodes collectively form a distributed array of antennas, providing a spatial diversity that can be exploited for reliable communication. Space-time codes (STC) [3], originally developed for point-topoint multiple-input-multiple-output (MIMO) communication,

Manuscript received December 26, 2013; revised May 24, 2014 and September 10, 2014; accepted September 14, 2014. Date of publication September 23, 2014; date of current version October 17, 2014. This work was supported in part by the Ministry of Science and Technology, Taiwan, under Grant MOST 103-2221-E-001-027. The associate editor coordinating the review of this paper and approving it for publication was M. Valenti.

S.-E. Chiu is with the Department of Electrical and Computer Engineering, University of California, San Diego, CA 95616 USA (e-mail: suchiu@eng. ucsd.edu).

F.-T. Chien is with the Department of Electronics Engineering, National Chiao Tung University, Hsinchu 300, Taiwan (e-mail: ftchien@mail. nctu.edu.tw).

R. Y. Chang is with the Research Center for Information Technology Innovation, Academia Sinica, Taipei 115, Taiwan (e-mail: rchang@ citi.sinica.edu.tw).

Color versions of one or more of the figures in this paper are available online at http://ieeexplore.ieee.org.

Digital Object Identifier 10.1109/TCOMM.2014.2359874 have been designed for cooperative relay networks with distributed antennas under frequency-flat [4]-[6] and frequencyselective [7], [8] fading environments to realize this spatial diversity. In a frequency-selective fading environment, an additional frequency diversity is created from the multipath channel fading, which motivates the development of distributed space-frequency codes (SFC) [7], [8]. Li et al. [7] proposed a distributed SFC for decode-and-forward (DF) relay systems employing orthogonal frequency-division multiplexing (OFDM) modulation. Perfect decoding was assumed at the relays. Seddik and Liu [8] studied the design of distributed SFC for both amplify-and-forward (AF) and DF relay systems with possible decoding errors at the relays.

When taking into account decoding imperfection at the relays, having all relays always participate in forwarding the source's information may not be the best strategy. It was shown [9], [10] that if each relay always participates in simple DF cooperative relaying regardless of whether its decoding was correct or not, the system may not achieve full diversity in distributed relay systems due to error propagation. Smart signal processing at the relays [8], [11], [12] or decoding schemes at the destination [13], [14] were therefore proposed to attain full diversity for the DF relaying systems. In particular, censoring was proposed [8], [11], [12] to be implemented at each relay to assess the relay's ability to decode, determine its follow-up action (cooperate or not), and thereby improve the performance and reliability of DF relaying. With censoring, essentially some form of error detection (explicit or implicit) is performed at each relay, which can be cyclic redundancy check (CRC)-based [8] (explicit) or signal-to-noise ratio (SNR)-based [11], [12] (implicit), to assess each relay's decoding ability before it reencodes and re-transmits to the destination. Perfect censoring, i.e., there is a perfect agreement between the censoring result and the actual state of decoding at each relay, was considered in [8], where the relay censoring is based on the CRC and the destination needs to know the relay decoding status to facilitate its own decoding. Relay censoring based on implementing a CRC code at the relay was also studied in [15] and [16]. Onat et al. [11], [12] proposed an SNR threshold-based relay censoring scheme for single-relay networks with binary phase shift keying (BPSK) modulation. Imperfect censoring was considered in [11] and [12], and the error propagation effect as a result of the relays decoding in error and forwarding an erroneous message due to imperfect censoring was addressed by designing an optimal censoring threshold to minimize the end-to-end bit error rate (BER). The asymptotic BER analysis 
in [12] showed that a full diversity of order two can be achieved in a single-relay network.

Perfect instantaneous channel state information (CSI) was assumed in the aforementioned work [4]-[8], [11], [12] to enable coherent detection at the destination. To obtain perfect CSI, an expensive effort is required to perform accurate channel estimation, which is challenging in general and especially in multi-hop wireless networks and/or fast-fading environments. Therefore, there has been much interest in communications that do not require the instantaneous CSI, which has been termed as noncoherent communications in [10] and [17]-[23]. Noncoherent communication in flat-fading MIMO systems was studied by Hochwald and Marzetta [17], where unitary STC was proposed to achieve the same diversity order as in coherent communication. The existence of such unitary STC and their construction were investigated by Tarokh and Kim [18], where an elegant geometric interpretation of ML decoding with unitary modulation was also presented. Noncoherent space-frequency coded MIMO-OFDM systems were studied by Borgmann and Bolcskei [19], where the diversity of the noncoherent MIMO-OFDM system was shown identical to its coherent counterpart.

Besides point-to-point communication, noncoherent communication was also studied in the context of wireless relay networks, e.g., for AF relaying [20]-[22] and DF relaying [10], [23], [24]. For AF relaying, Souryal [20] proposed a noncoherent generalized likelihood ratio test (GLRT)-based receiver that requires local noise power information but not instantaneous/statistical CSI, and showed that near secondorder diversity can be achieved for the single-relay scenario. Lin et al. [21] proposed a noncoherent GLRT-based sequence detector that requires neither noise power information nor CSI, and demonstrated that near full diversity can be achieved for three special relaying scenarios with at most two relays. Farhadi and Beaulieu [22] developed a completely noncoherent receiver based on maximum energy selection (MES) and showed that the noncoherent MES detection can promise full spatial diversity with any number of relays. For DF relaying, Chen and Laneman [10] showed that, with noncoherent detection at the destination and with multiple relays that always transmit, the diversity order is only about half of the number of relays due to error propagation. Chang et al. [23] proposed a noncoherent orthogonal space-time block coded (OSTBC) OFDM scheme to enable simultaneous transmission (as opposed to orthogonal transmission in [20]-[22]) and showed that the proposed distributed OSTBC scheme can achieve full spatial diversity when perfect decoding at the relays is established. Valenti et al. [24] studied the noncoherent receiver design that does not require the phase information for physical-layer network coded relay systems for the cases of known and unknown channel amplitudes.

\section{A. Motivations}

Noncoherent communication in relay networks has not been as well studied for frequency-selective fading channels (e.g., [23]) as for flat fading channels (e.g., [10], [20]-[22]). In particular, the combination of noncoherent distributed SFC and relay censoring for DF relaying has not been investigated. We are therefore motivated to conduct an analytical study of the maximum achievable diversity in noncoherent distributed SFC systems with multiple censored DF relays by considering both perfect and imperfect censoring. The design and analysis of noncoherent distributed SFC systems with relay censoring are not direct extensions from point-to-point communication systems (e.g., [19]) because: 1) For DF relaying with multiple censored relays, the relay decoding status information (i.e., whether a relay has successfully decoded and will participate in cooperative transmission) is not available at the destination especially in noncoherent settings; and 2) The error propagation effect due to censoring errors dramatically complicates the diversity analysis.

\section{B. Contributions}

In this paper, we perform the diversity analysis of a noncoherent, distributed space-frequency coded OFDM-based DF relaying system under frequency-selective fading environments. We consider a network comprised of a single source, multiple relays, and a single destination. Similar to [8], [11], and [25], censoring is performed before each relay decodes and forwards the source's information toward the destination. We derive the achievable diversity analytically for both cases of perfect and imperfect relay censoring. The main contributions of this paper are summarized below:

- With perfect relay censoring, we consider cases with and without partial information of channel statistics and relay decoding status at the destination. When partial information is available, we show that a diversity of order $R \cdot \min \left\{L_{s}, L\right\}$ can be achieved with the ML decoder, where $R$ is the number of cooperating relays, and $L_{s}$ and $L$ are the channel order (number of delay paths) between the source and each relay and between each relay and the destination, respectively. When partial information is not available, i.e., a completely noncoherent receiver, we show that both the ML decoder and a proposed suboptimal correlator-based decoder can still yield the same diversity of order $R \cdot \min \left\{L_{s}, L\right\}$. The correlator-based decoder was proposed for analytical tractability to verify the diversity result for the (completely noncoherent) ML decoder as well as for its own merits, since 1) it does not require instantaneous/statistical CSI and the relay decoding status information, and 2) it allows a simple closed-form decoding rule at the destination. The derived diversity results are numerically verified.

- With imperfect relay censoring, i.e., taking into account the possible mismatch between the censoring result and the actual state of decoding at each relay, we characterize the relationship between the censoring error probabilities and the achievable diversity of the system analytically. We show that when the likelihood of two types of censoring errors increases, the suboptimal correlator-based decoder may not attain the full diversity of order $R \cdot \min \left\{L_{s}, L\right\}$. Numerical results demonstrate an increased performance gap between the ML decoder and the correlator-based decoder with imperfect relay censoring. The results suggest 


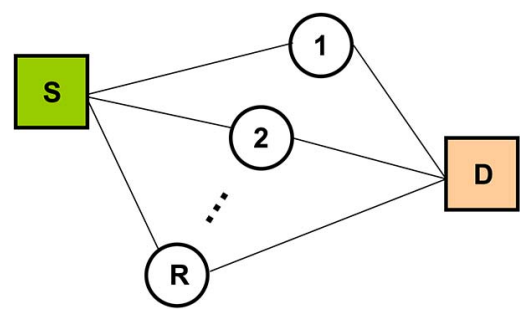

Fig. 1. A two-hop wireless relay system with a single source, $R$ relays, and a single destination.

that the noncoherent distributed system can offer a comparable diversity as the conventional MIMO-OFDM system provided that relay censoring is carefully implemented.

The outline of this paper is as follows. Section II presents the system model. The diversity analysis is conducted in Section III with perfect relay censoring and Section IV with imperfect relay censoring. Simulation results and discussions are presented in Section V. Conclusion is given in Section VI.

Notations: $\operatorname{diag}\left\{\mathbf{d}_{1}, \ldots, \mathbf{d}_{D}\right\}$ denotes the diagonal matrix with $\mathbf{d}_{1}, \ldots, \mathbf{d}_{D}$ as the diagonal entries where $\mathbf{d}_{i}, 1 \leq i \leq D$, can be a vector or scalar. $f_{1}(\rho) \doteq f_{2}(\rho)$ represents the diversity equivalence relation, i.e., $f_{1}$ and $f_{2}$ have the same exponent, or mathematically $\lim _{\rho \rightarrow \infty} \frac{\log f_{1}(\rho)}{\log \rho}=\lim _{\rho \rightarrow \infty} \frac{\log f_{2}(\rho)}{\log \rho}$.

\section{SySTEM MOdEL}

Consider a two-hop wireless relay network consisting of a single source, $R$ relays, and a single destination, as depicted in Fig. 1. All nodes are each equipped with a single antenna. The information is transmitted from the source to the destination in two time slots via the cooperative DF communication protocol. In Slot I, the source node broadcasts the information message to the relays. We assume that in Slot I there is no direct link from the source to the destination for ease of analytical development, similar to [8]. In Slot II, with the source node silent, each relay first adopts a censoring scheme (e.g., an SNR threshold-based approach [11]) to assess its decoding ability and determine whether it should participate in cooperative transmission in Slot II. Only relays that pass the censoring (e.g., the received SNR is greater than a threshold) will decode the received signals and then cooperatively and concurrently forward the re-encoded signals toward the destination. Depending on the censoring result and the actual state of decoding at each relay, we have four possibilities for each relay:

1) Useful relay: The relay passes the censoring and thus will decode and forward in Slot II. The relay indeed decodes correctly.

2) Harmful relay: The relay passes the censoring and thus will decode and forward in Slot II. The relay however decodes incorrectly.

3) Useless relay: The relay fails the censoring and thus will not decode and forward in Slot II. However, the relay would decode correctly if decoding were performed.

4) Controlled relay: The relay fails the censoring and thus will not decode and forward in Slot II. Indeed, the relay would decode incorrectly if decoding were performed.
We call a relay in the first two cases an active node and in the latter two cases a silent node. The probability of each of the four events is determined by the relay decoding error probability $p_{s}^{r}$ as well as the two types of relay censoring errors:

$$
\begin{aligned}
& p_{0 \mid 1}^{r} \triangleq \mathrm{P}\left(\text { relay } \mathrm{R}_{r} \text { is active } \mid \mathrm{R}_{r} \text { decodes incorrectly }\right), \\
& p_{1 \mid 0}^{r} \triangleq \mathrm{P}\left(\text { relay } \mathrm{R}_{r} \text { is silent } \mid \mathrm{R}_{r} \text { decodes correctly }\right),
\end{aligned}
$$

where the superscript $r(r=1, \ldots, R)$ indicates that these probabilities are relay-dependent and are specific to the $r$ th relay $\mathrm{R}_{r}$. The probabilities $1-p_{1 \mid 0}^{r}, p_{0 \mid 1}^{r}, p_{1 \mid 0}^{r}$, and $1-p_{0 \mid 1}^{r}$ are related to the four events above for relay $\mathrm{R}_{r}$ (i.e., useful, harmful, useless, and controlled, in that order), respectively. The probability $p_{s}^{r}$ that relay $\mathrm{R}_{r}$ erroneously decodes the transmitted signal in Slot I depends on the coding/decoding scheme and the channel condition between source and relay $\mathrm{R}_{r}$. The values of $p_{0 \mid 1}^{r}$ and $p_{1 \mid 0}^{r}$ depend on the censoring scheme employed at $\mathrm{R}_{r}$. For example, in the SNR threshold-based relay censoring considered in [11] and [12] with flat Rayleigh faded sourcerelay channel and BPSK signaling, a closed-form expression of $p_{0 \mid 1}^{r}$ can be derived using [12, Eqs. (4) and (6)], the error decoding probability $p_{s}^{r}$, and $\mathrm{P}$ (relay $\mathrm{R}_{r}$ is active). Then, $p_{1 \mid 0}^{r}$ can be obtained from $p_{0 \mid 1}^{r}$ by the Bayes' rule.

All relay nodes in the network employ OFDM-based transmission with $N$ subcarriers. The baseband frequency-selective fading channel between the $r$ th relay and the destination node has $L$ independent delay paths $h_{r}(l), 0 \leq l \leq L-1$. The channel gain $h_{r}(l)$ is modeled as complex Gaussian random variable (statistically independent across both $r$ and $l$ ) with zero mean and variance $\sigma_{r, l}^{2}$, where $\sum_{l=0}^{L-1} \sigma_{r, l}^{2}=1,1 \leq r \leq R$ for channel power normalization. In Slot II transmission, an active relay node re-encodes its decoded message using a mapping, i.e., space-frequency coding. Let the constellation alphabet with size $K$ used in Slot I be $\mathfrak{S}=\left\{\mathbf{s}_{0} \mathbf{s}_{1} \cdots \mathbf{s}_{K-1}\right\}$. Further, let the decoded signal at the $r$ th active relay be $\hat{\mathbf{s}}^{r}$. Suppose that the $r$ th relay has decoded the received signal as $\hat{\mathbf{s}}^{r}=\mathbf{s}_{k}$. Then, it will re-encode the message into an $N \times 1$ OFDM symbol vector $\mathbf{c}_{k}^{r}$ for transmission according to the mapping $\mathbf{s}_{k} \mapsto \mathbf{c}_{k}^{r}$. The superscript $r$ indicates that different relays may re-encode the message differently. Let $\mathbf{C}_{i}=\left[\begin{array}{cccc}\mathbf{c}_{i}^{1} \mathbf{c}_{i}^{2} & \cdots & \mathbf{c}_{i}^{R}\end{array}\right]$ represent the codeword matrix constructed when all relays have $\mathbf{s}_{i}$ as the decoded message. The codebook $\mathcal{C}$ for Slot II transmission is therefore defined as $\mathcal{C}=\left\{\mathbf{C}_{0} \mathbf{C}_{1} \cdots \mathbf{C}_{K-1}\right\}$. In Slot II, the destination receives the sum of signals from all active relays. The received signal $\mathbf{r}_{d}$ at the destination node after Fast Fourier Transform (FFT) can be expressed as

$$
\mathbf{r}_{d}=\sqrt{\rho} \sum_{r: \text { active }} \mathbf{H}_{r} \mathbf{c}^{r}+\mathbf{n},
$$

where $\rho$ is the power scaling factor for each relay, $\mathbf{c}^{r} \in$ $\left\{\mathbf{c}_{0}^{r}, \ldots, \mathbf{c}_{K-1}^{r}\right\}$ is the transmitted codeword from the $r$ th relay, $\mathbf{H}_{r}$ is a diagonal matrix with the $n$th diagonal entry $\left(\mathbf{H}_{r}\right)_{n n}$ being the frequency response of the channel gain from the $r$ th relay to the destination at the $n$th subcarrier, and $\mathbf{n} \sim \mathcal{C N}\left(\mathbf{0}, \mathbf{I}_{N}\right)$ is complex Gaussian noise vector. The matrix $\mathbf{H}_{r}$ and the channel gain are related according to $\mathbf{H}_{r}=\sum_{l=0}^{L-1} h_{r}(l) \mathbf{D}^{l}$, 
where $\mathbf{D}=\operatorname{diag}\left\{1, e^{-j 2 \pi \frac{1}{N}}, \ldots, e^{-j 2 \pi \frac{N-1}{N}}\right\}$. Substituting $\mathbf{H}_{r}$ into (1) and reordering the summation give

$$
\mathbf{r}_{d}=\sqrt{\rho} \sum_{l=0}^{L-1} \mathbf{D}^{l} \sum_{r: \text { active }} h_{r}(l) \mathbf{c}^{r}+\mathbf{n}
$$

To characterize the relays' activities, we define a relay status matrix $\mathbf{S}_{m}=\operatorname{diag}\left\{S_{m, 1}, \ldots, S_{m, R}\right\}$ where

$$
S_{m, r}= \begin{cases}1, & \text { if } \mathrm{R}_{r} \text { is active and has decoded as } \hat{\mathbf{s}}^{r}=\mathbf{s}_{m}, \\ 0, & \text { otherwise. }\end{cases}
$$

The $S_{m, r}$ signifies the activity of the $r$ th relay with respect to the $m$ th information message.

Assuming that the transmitted signal from the source node in Slot $\mathrm{I}$ is $\mathbf{s}_{i}$, we have

$$
\mathbf{r}_{d}=\sqrt{\rho} \sum_{l=0}^{L-1} \mathbf{D}^{l}\left(\mathbf{C}_{i} \mathbf{S}_{i} \mathbf{h}(l)+\sum_{m \neq i}^{K-1} \mathbf{C}_{m} \mathbf{S}_{m} \mathbf{h}(l)\right)+\mathbf{n},
$$

with $\mathbf{h}(l)=\left[h_{1}(l) h_{2}(l) \ldots h_{R}(l)\right]^{T}$. Since the relay status matrix $\mathbf{S}_{i}$ will nullify the terms that correspond to the relays that either did not participate or did not decode correctly as $\mathbf{s}_{i}$, we can rewrite $\mathbf{r}_{d}$ as

$$
\mathbf{r}_{d}=\sqrt{\rho} \mathbf{E}_{i} \hat{\boldsymbol{S}}_{i} \mathbf{h}+\sqrt{\rho} \sum_{j \neq i}^{K-1} \mathbf{E}_{j} \hat{\boldsymbol{S}}_{j} \mathbf{h}+\mathbf{n} .
$$

where $\mathbf{E}_{i}=\left[\mathbf{C}_{i} \mathbf{D C}_{i} \cdots \mathbf{D}^{L-1} \mathbf{C}_{i}\right]$ is the pseudo-codeword matrix [19] associated with $\mathbf{C}_{i}, \hat{\boldsymbol{S}}_{i}=\mathbf{I}_{L} \otimes \mathbf{S}_{i}$ is the stacked relay status matrix with $\mathbf{I}_{L}$ being the identity matrix of order $L$ and $\otimes$ being the Kronecker product, and $\mathbf{h}=\left[\mathbf{h}^{T}(0) \cdots \mathbf{h}^{T}(L-\right.$ 1) $]^{T}$ is the stacked channel vector. Note that the second term in (3) corresponds to the interference (error propagation) contributed by the harmful relays.

Definition: The diversity order is defined as $-\lim _{\rho \rightarrow \infty} \frac{\log \operatorname{PEP}(\rho)}{\log \rho}$, i.e., the asymptotic exponent in $\rho$ of the pairwise error probability $\operatorname{PEP}(\rho)$.

\section{DiVERSity ANALYSiS With PERFECT RELAy CENSORING}

In this paper, we analytically study the diversity order of the system when the receiver does not know the instantaneous CSI (h), i.e., noncoherent detection, by considering perfect (this section) and imperfect (Section IV) relay censoring. By perfect relay censoring we mean that there is a perfect agreement between the censoring result and the actual state of decoding at each relay [6], [8]; specifically, $p_{1 \mid 0}^{r}=0$ and $p_{0 \mid 1}^{r}=0$ for all $r=1, \ldots, R$, i.e., the cases of useless and harmful relays are precluded. The analysis in this section is of theoretical usefulness, which allows us to explore the core ideas of this work. We will relax the perfect censoring assumption later in Section IV. As a result of this consideration we have $\hat{\boldsymbol{S}}_{m}=\mathbf{0}$ for $m \neq i$ when the transmitted message from the source is $\mathbf{s}_{i}$. Thus,

$$
\mathbf{r}_{d}=\sqrt{\rho} \mathbf{E} \hat{\mathbf{S h}}+\mathbf{n}
$$

where $\mathbf{E} \in\left\{\mathbf{E}_{0}, \ldots, \mathbf{E}_{K-1}\right\}$ and $\hat{\boldsymbol{S}}=\mathbf{I}_{L} \otimes \mathbf{S}$ with $\mathbf{S}=\operatorname{diag}\left\{S_{1}\right.$, $\left.\ldots, S_{R}\right\}$ and

$$
S_{r}= \begin{cases}1, & \text { if } \mathrm{R}_{r} \text { is active } \\ 0, & \text { otherwise }\end{cases}
$$

for $1 \leq r \leq R$. Our analysis is conducted for two separate cases depending on whether partial information is available at the destination. In Section III-A, we consider the case where the destination has the (long-term) channel statistics $\left(\sigma_{r, l}^{2}, \forall r, l\right)$ and the relay decoding status $\mathbf{S}$ information, as in [8]. In Section III-B, we consider the previously unexamined case where the destination has neither the channel statistics nor the relay censoring information, i.e., a completely noncoherent receiver.

\section{A. Noncoherent Receiver With Known Channel Statistics and Relay Status}

1) Maximum Likelihood Decoding: We first evaluate the minimum achievable error probability with ML decoding. With known channel statistics and relay decoding status, the likelihood function $p\left(\mathbf{r}_{d} \mid \mathbf{C}_{i}, \hat{\mathbf{S}}\right)$ is multivariate complex Gaussian. Given that the transmitted codeword matrix is $\mathbf{C}_{i}$, the covariance matrix of $\mathbf{r}_{d}$ is $\boldsymbol{\Lambda}_{i}=\mathbf{I}_{N}+\rho \mathbf{E}_{i} \hat{\boldsymbol{S}} \boldsymbol{\Sigma}^{2} \mathbf{E}_{i}^{H}$, where $\boldsymbol{\Sigma}^{2} \triangleq$ $E\left[\mathbf{h} \mathbf{h}^{H}\right]=\operatorname{diag}\left\{\boldsymbol{\sigma}_{0}^{2}, \ldots, \boldsymbol{\sigma}_{L-1}^{2}\right\}$ is the covariance matrix of $\mathbf{h}$ with $\boldsymbol{\sigma}_{l}^{2}=\left[\sigma_{1, l}^{2} \sigma_{2, l}^{2} \ldots \sigma_{R, l}^{2}\right]^{T}$ for $l=0, \ldots, L-1$. Note that we have exploited the fact that $\hat{\boldsymbol{S}}^{2}=\hat{\boldsymbol{S}}$ and $\boldsymbol{\Sigma}^{2}$ is diagonal in the expression of $\boldsymbol{\Lambda}_{i}$. The conditional density of the received signal is then given by

$$
p\left(\mathbf{r}_{d} \mid \mathbf{C}_{i}, \hat{\boldsymbol{S}}\right)=\frac{1}{\pi^{N} \operatorname{det}\left(\boldsymbol{\Lambda}_{i}\right)} \exp \left(-\mathbf{r}_{d}^{H} \boldsymbol{\Lambda}_{i}^{-1} \mathbf{r}_{d}\right) .
$$

Consequently, we have the ML decoding rule $\hat{\boldsymbol{C}}_{\mathrm{ML}}=$ $\arg \min _{\mathbf{C}_{i} \in \mathcal{C}}\left(\mathbf{r}_{d}^{H} \boldsymbol{\Lambda}_{i}^{-1} \mathbf{r}_{d}+\ln \operatorname{det} \boldsymbol{\Lambda}_{i}\right)$.

We restrict ourselves to the case of a unitary codebook, i.e., $\mathbf{E}_{i}^{H} \mathbf{E}_{i}=\mathbf{I}_{R L}$ for all $i$, for analytical tractability. This consideration first appeared in [17] and has since been widely adopted in the design and analysis of STC and SFC systems [5], [8], [19]. Applying the matrix inversion lemma to $\boldsymbol{\Lambda}_{i}^{-1}$, the $\mathrm{ML}$ decoding rule is simplified as

$$
\hat{\mathbf{C}}_{\mathrm{MLA}}=\underset{\mathbf{C}_{i} \in \mathcal{C}}{\arg \max }\left(\mathbf{r}_{d}^{H} \mathbf{E}_{i} \hat{\boldsymbol{S}} \boldsymbol{\Sigma}^{2}\left(\mathbf{I}_{R L}+\rho \hat{\boldsymbol{S}} \boldsymbol{\Sigma}^{2}\right)^{-1} \mathbf{E}_{i}^{H} \mathbf{r}_{d}\right),
$$

where the subscript MLA represents the ML decoding rule considered in Section III-A (to distinguish from the one considered in Section III-B). The ML decoding rule here requires knowledge of the relay decoding status $\hat{\mathbf{S}}$ and the channel statistics $\Sigma^{2}$ at the destination node.

2) Conditional Pairwise Error Probability: Based on the ML decoding in (5), we derive the pairwise error probability (PEP) of deciding on $\mathbf{C}_{j}$ at the destination given that $\mathbf{C}_{i}$ was actually transmitted. The PEP conditioned on the relay status matrix $\hat{\mathbf{S}}$ is given by

$\mathrm{P}\left(\mathbf{C}_{i} \rightarrow \mathbf{C}_{j} \mid \mathbf{C}_{i}, \hat{\boldsymbol{S}}\right)=\mathrm{P}\left(v>0 \mid \mathbf{C}_{i}, \hat{\boldsymbol{S}}\right) \leq E\left[e^{s v} \mid \mathbf{C}_{i}, \hat{\boldsymbol{S}}\right], \forall s>0$, 
where the inequality follows from the Chernoff bound and $v=$ $\mathbf{r}_{d}^{H}\left(\mathbf{E}_{j} \hat{\boldsymbol{S}} \boldsymbol{\Sigma}^{2} \boldsymbol{\Theta}^{-1} \mathbf{E}_{j}^{H}-\mathbf{E}_{i} \hat{\boldsymbol{S}} \boldsymbol{\Sigma}^{2} \boldsymbol{\Theta}^{-1} \mathbf{E}_{i}^{H}\right) \mathbf{r}_{d}$ with $\boldsymbol{\Theta}=\mathbf{I}_{R L}+$ $\rho \hat{\boldsymbol{S}} \boldsymbol{\Sigma}^{2}$. Following a similar algebraic approach in [19], a diversity preserving bound can be obtained as

$$
\mathrm{P}\left(\mathbf{C}_{i} \rightarrow \mathbf{C}_{j} \mid \mathbf{C}_{i}, \hat{\boldsymbol{S}}\right)<\left(\frac{\rho}{4}\right)^{-n_{\mathbf{S}_{i}} L} \prod_{n=0}^{n_{\mathbf{S}_{i}} L-1} \lambda_{n}^{-1}\left(\hat{\boldsymbol{S}} \boldsymbol{\Sigma}^{2} \mathbf{Q}\right),
$$

where $n_{\mathbf{S}_{i}}=\sum_{r=1}^{R} S_{r}$ is the number of active relays with decoded $\mathbf{s}_{i}, \lambda_{n}\left(\hat{\boldsymbol{S}} \boldsymbol{\Sigma}^{2} \mathbf{Q}\right)$ represents the $n$th eigenvalue of $\hat{\boldsymbol{S}} \boldsymbol{\Sigma}^{2} \mathbf{Q}$, and $\mathbf{Q}=\mathbf{I}_{R L}-\mathbf{E}_{j}^{H} \mathbf{E}_{i} \mathbf{E}_{i}^{H} \mathbf{E}_{j}$. In (7), the decay rate of the conditional PEP scales with $\rho^{-n_{\mathbf{S}_{i}} L}$ when $\mathbf{Q}$ is full rank [19].

3) Average Pairwise Error Probability: We obtain the average PEP at the destination node by averaging over all $\hat{\boldsymbol{S}}$. Diversity order can be derived from the obtained average PEP. With perfect relay censoring, the state of the $r$ th relay node $S_{r}$ is a Bernoulli random variable, i.e., $S_{r}=0$ with probability $p_{s}^{r}$ and $S_{r}=1$ with probability $1-p_{s}^{r}$. Hereafter, we consider that the relay decoding error probability $p_{s}^{r}$ scales with $\rho^{-L_{s}}$ (i.e., $p_{s}^{r} \doteq \rho^{-L_{s}}$ ), where $L_{s}$ is the number of delay paths between the source and each relay. This scaling law is achievable at each relay through proper coding schemes. To analyze the diversity order of the system, we first provide the following lemma.

Lemma: Let $f(\rho)=\sum_{i=1}^{n} \alpha_{i} \rho^{-l_{i}}$, where $l_{1} \leq l_{2} \leq \ldots \leq$ $l_{n}$ are nonnegative integers and $\alpha_{i}$ is a positive constant irrelevant of $\rho$ for $i=1, \ldots, n$. Then, we have $f(\rho) \doteq \rho^{-l_{1}}$.

Proof: As $\rho \rightarrow \infty$, we have

$$
\begin{aligned}
\frac{1}{\log \rho} \log f(\rho) & =\frac{1}{\log \rho}\left(\log \rho^{-l_{1}}+\log \sum_{i=1}^{n} \alpha_{i} \rho^{-\left(l_{i}-l_{1}\right)}\right) \\
& \rightarrow \frac{1}{\log \rho} \log \rho^{-l_{1}}
\end{aligned}
$$

where the relation $l_{i} \geq l_{1}, i=1, \ldots, n$, is exploited in deriving the asymptotic behavior.

Lemma 1 states that if the average PEP assumes the form of $f(\rho)$ in Lemma 1, the diversity order is determined by the dominant exponent.

Proposition 1: With perfect relay censoring, the maximum achievable diversity order is $R \cdot \min \left\{L_{s}, L\right\}$ with the noncoherent ML decoder in (5).

Proof: The average PEP at the destination can be expressed as

$$
\mathrm{P}\left(\mathbf{C}_{i} \rightarrow \mathbf{C}_{j} \mid \mathbf{C}_{i}\right)=\sum_{\hat{\boldsymbol{S}}} \mathrm{P}\left(\hat{\boldsymbol{S}} \mid \mathbf{C}_{i}\right) \mathrm{P}\left(\mathbf{C}_{i} \rightarrow \mathbf{C}_{j} \mid \mathbf{C}_{i}, \hat{\boldsymbol{S}}\right)
$$

where $\mathrm{P}\left(\mathbf{C}_{i} \rightarrow \mathbf{C}_{j} \mid \mathbf{C}_{i}, \hat{\boldsymbol{S}}\right)$ is upper bounded in (7), and $\mathrm{P}\left(\hat{\boldsymbol{S}} \mid \mathbf{C}_{i}\right)=\prod_{j \in \mathcal{R}_{u \mid i}}\left(1-p_{s}^{j}\right) \cdot \prod_{k \in \mathcal{R}_{s \mid i}} p_{s}^{k}$ with $\mathcal{R}_{u \mid i}$ being the set of useful relays (with cardinality $n_{\mathbf{S}_{i}}$ ) and $\mathcal{R}_{s \mid i}$ the set of silent relays (with cardinality $R-n_{\mathbf{S}_{i}}$ ) given that the transmitted message in Slot $\mathrm{I}$ is $\mathbf{s}_{i}$. There is no harmful relay due to perfect relay censoring. The average PEP is

$$
\begin{aligned}
& \mathrm{P}\left(\mathbf{C}_{i} \rightarrow \mathbf{C}_{j} \mid \mathbf{C}_{i}\right) \\
& \doteq \sum_{\hat{\mathbf{s}}}\left(\prod_{j \in \mathcal{R}_{u \mid i}}\left(1-p_{s}^{j}\right)\right) \cdot\left(\prod_{k \in \mathcal{R}_{s \mid i}} p_{s}^{k}\right) \cdot \gamma_{n_{\mathbf{S}_{i}}} \rho^{-n_{\mathbf{S}_{i}} L},
\end{aligned}
$$

where the diversity equivalence follows from the diversity preserving bound in (7), and $\gamma_{n_{\mathbf{S}_{i}}} \triangleq 4^{n_{\mathbf{S}_{i}} L} \prod_{n=0}^{n_{\mathbf{S}_{i}} L-1} \lambda_{n}\left(\hat{\boldsymbol{S}}^{-1} \boldsymbol{\Sigma}^{2} \mathbf{Q}\right)$ is irrelevant to $\rho$. According to Lemma 1, to obtain the diversity order we only need to find the dominant exponent and can discard $\gamma_{n_{\mathrm{s}_{i}}}$ in (9). As $\rho \rightarrow \infty$ and with $p_{s}^{r} \doteq \rho^{-L_{s}}$, we have

$$
\begin{aligned}
-\frac{\log }{\log \rho} & \prod_{k \in \mathcal{R}_{s \mid i}} p_{s}^{k} \cdot \rho^{-n_{\mathbf{S}_{i}} L} \\
& \rightarrow \frac{-1}{\log \rho}\left(\left(\sum_{k \in \mathcal{R}_{s \mid i}} \log p_{s}^{k}\right)+\log \rho^{-n_{\mathbf{S}_{i}} L}\right) \\
& =\left(R-n_{\mathbf{S}_{i}}\right) L_{s}+n_{\mathbf{S}_{i}} L .
\end{aligned}
$$

From (9), (10), and Lemma 1, it follows that the diversity order is $\min _{0 \leq n_{\mathbf{S}_{i}} \leq R}\left(\left(R-n_{\mathbf{S}_{i}}\right) L_{s}+n_{\mathbf{S}_{i}} L\right)=R \cdot \min \left\{L_{s}, L\right\}$.

\section{B. Completely Noncoherent Receiver}

1) Maximum Likelihood Decoding and Correlator-Based Decoding: To obtain the likelihood function without knowledge of $\hat{\boldsymbol{S}}$, we take the expectation of (4) over all possible $\hat{\boldsymbol{S}}$, which yields a Gaussian mixture $p\left(\mathbf{r}_{d} \mid \mathbf{C}_{i}\right)=$ $\sum_{\hat{\mathbf{S}}} \mathrm{P}\left(\hat{\mathbf{S}} \mid \mathbf{C}_{i}\right) p\left(\mathbf{r}_{d} \mid \mathbf{C}_{i}, \hat{\mathbf{S}}\right)$. The optimal ML decoding corresponds to

$$
\hat{\mathbf{C}}_{\mathrm{MLB}}=\underset{\mathbf{C}_{i} \in \mathcal{C}}{\arg \max } p\left(\mathbf{r}_{d} \mid \mathbf{C}_{i}\right),
$$

where the subscript MLB represents the ML decoding rule considered in Section III-B. The error probability and diversity analysis based on (11) is analytically intractable since there exists no closed-form decision rule in (11). To overcome this obstacle, we consider a low-complexity, completely noncoherent receiver. Specifically, we let $\hat{\boldsymbol{S}}=\mathbf{I}_{R L}$ (i.e., no relay decoding status is needed) and $\boldsymbol{\Sigma}^{2}=\mathbf{I}_{R L}$ (i.e., no statistical CSI is needed) to yield a suboptimal correlator-based decoder which exploits only the correlation structure of the codeword matrices, i.e.,

$$
\hat{\mathbf{C}}_{\text {subopt }}=\underset{\mathbf{C}_{i} \in \mathcal{C}}{\arg \max }\left(\mathbf{r}_{d}^{H} \mathbf{E}_{i} \mathbf{E}_{i}^{H} \mathbf{r}_{d}\right) .
$$

Note that this suboptimal decoder approximates the ML decoder in (11) well in the high SNR regime since all relays with a proper censoring mechanism tend to be useful relays. In what follows, we first analyze the achievable diversity order with the suboptimal decoder and then deduce from the result about the achievable diversity order with the ML decoding in (11).

2) Conditional Pairwise Error Probability: The conditional PEP based on the decoding rule in (12) can be shown to be upper bounded by

$\mathrm{P}\left(\mathbf{C}_{i} \rightarrow \mathbf{C}_{j} \mid \mathbf{C}_{i}, \hat{\boldsymbol{S}}\right)=\mathrm{P}\left(w>0 \mid \mathbf{C}_{i}, \hat{\boldsymbol{S}}\right) \leq E\left[e^{s w} \mid \mathbf{C}_{i}, \hat{\boldsymbol{S}}\right]$,

$$
\forall s \geq 0,
$$


where $w \triangleq \mathbf{r}_{d}^{H} \mathbf{E}_{j} \mathbf{E}_{j}^{H} \mathbf{r}_{d}-\mathbf{r}_{d}^{H} \mathbf{E}_{i} \mathbf{E}_{i}^{H} \mathbf{r}_{d}$. Substituting $\quad \mathbf{r}_{d}=$ $\sqrt{\rho} \mathbf{E}_{i} \hat{\boldsymbol{S}} \mathbf{h}+\mathbf{n}$ into $w$ and conditioning on the channel yield

$$
\begin{aligned}
E\left[e^{s w} \mid \mathbf{C}_{i}, \hat{\boldsymbol{S}}, \mathbf{h}\right] & \\
= & \exp \left(s \rho \mathbf{h}^{H} \hat{\boldsymbol{S}}\left(\mathbf{E}_{i}^{H} \mathbf{E}_{j} \mathbf{E}_{j}^{H} \mathbf{E}_{i}-\mathbf{I}_{R L}\right) \hat{\boldsymbol{S}} \mathbf{h}\right) \\
\times & {\left[\exp \left(s \mathbf{n}^{H}\left(\mathbf{E}_{j} \mathbf{E}_{j}^{H}-\mathbf{E}_{i} \mathbf{E}_{i}^{H}\right) \mathbf{n}\right)\right.} \\
& \cdot \exp \left(2 s \Re\left\{\sqrt{\rho} \mathbf{h}^{H} \hat{\boldsymbol{S}} \mathbf{E}_{i}^{H}\left(\mathbf{E}_{j} \mathbf{E}_{j}^{H}-\mathbf{I}_{N}\right) \mathbf{n}\right\}\right) \\
& \left.\mid \mathbf{C}_{i}, \hat{\boldsymbol{S}}, \mathbf{h}\right] \\
\leq & \exp \left(s \rho \mathbf{h}^{H} \hat{\boldsymbol{S}}\left(\mathbf{E}_{i}^{H} \mathbf{E}_{j} \mathbf{E}_{j}^{H} \mathbf{E}_{i}-\mathbf{I}_{R L}\right) \hat{\boldsymbol{S}} \mathbf{h}\right) \\
\times & \left(E\left[\exp \left(2 s \mathbf{n}^{H}\left(\mathbf{E}_{j} \mathbf{E}_{j}^{H}-\mathbf{E}_{i} \mathbf{E}_{i}^{H}\right) \mathbf{n}\right) \mid \mathbf{C}_{i}, \hat{\boldsymbol{S}}, \mathbf{h}\right]\right)^{\frac{1}{2}} \\
\times & \left(E \left[\exp \left(4 s \Re\left\{\sqrt{\rho} \mathbf{h}^{H} \hat{\boldsymbol{S}} \mathbf{E}_{i}^{H}\left(\mathbf{E}_{j} \mathbf{E}_{j}^{H}-\mathbf{I}_{N}\right) \mathbf{n}\right\}\right)\right.\right. \\
& \left.\left.\mid \mathbf{C}_{i}, \hat{\boldsymbol{S}}, \mathbf{h}\right]\right)^{\frac{1}{2}}
\end{aligned}
$$

where $\Re\{\cdot\}$ denotes the real part of its argument, and the inequality follows from the Cauchy-Schwarz inequality. The first expectation in (15) is related to the MGF of a Hermitian quadratic form of complex Gaussian random variables, for which the closed-form solution can be found in [26]. The second expectation in (15) can be evaluated using the moment generating function (MGF) of a Gaussian random variable. Carrying out these two expectations we have

$$
\begin{aligned}
& E\left[e^{s w}\right.\left.\mid \mathbf{C}_{i}, \hat{\boldsymbol{S}}, \mathbf{h}\right] \\
& \leq \exp \left(\left(s-2 s^{2}\right) \rho \mathbf{h}^{H} \hat{\boldsymbol{S}}\left(\mathbf{E}_{i}^{H} \mathbf{E}_{j} \mathbf{E}_{j}^{H} \mathbf{E}_{i}-\mathbf{I}_{R L}\right) \hat{\boldsymbol{S}} \mathbf{h}\right) \\
& \quad \cdot \operatorname{det}^{-\frac{1}{2}}\left(\mathbf{I}_{N}-2 s\left(\mathbf{E}_{j} \mathbf{E}_{j}^{H}-\mathbf{E}_{i} \mathbf{E}_{i}^{H}\right)\right),
\end{aligned}
$$

where the bound is valid for any $s$ satisfying $0<s<\frac{1}{2}$ for a positive definite $\mathbf{I}_{N}-2 s\left(\mathbf{E}_{j} \mathbf{E}_{j}^{H}-\mathbf{E}_{i} \mathbf{E}_{i}^{H}\right)$.

Note that while it is possible to obtain the optimal $s$ that minimizes the bound, any $s$ satisfying $0<s<\frac{1}{2}$ is sufficient to provide a valid upper bound in (16). This is because any fixed $s$ within $0<s<\frac{1}{2}$ just constitutes a constant scaling factor in (16) and does not alter the exponent in $\rho$. Consequently, any legitimate $s$ will yield the same diversity order as in (18) and (19) shown later. We adopt $s=\frac{1}{3}$, which leads the determinant in (16) to be bounded by

$$
\operatorname{det}^{-\frac{1}{2}}\left(\mathbf{I}_{N}-\frac{2}{3}\left(\mathbf{E}_{j} \mathbf{E}_{j}^{H}-\mathbf{E}_{i} \mathbf{E}_{i}^{H}\right)\right) \leq\left(\frac{1}{3}\right)^{-\frac{N}{2}},
$$

where we have used the Weyl's inequality [27, Theorem 4.3.1] that $\lambda\left(\mathbf{E}_{j} \mathbf{E}_{j}^{H}-\mathbf{E}_{i} \mathbf{E}_{i}^{H}\right) \leq \lambda_{\max }\left(\mathbf{E}_{j} \mathbf{E}_{j}^{H}\right)-\lambda_{\min }\left(\mathbf{E}_{i} \mathbf{E}_{i}^{H}\right)=1$. Substituting (17) into (16) and then (16) into (13) leads to

$$
\begin{aligned}
\mathrm{P}(w & \left.>0 \mid \mathbf{C}_{i}, \hat{\boldsymbol{S}}, \mathbf{h}\right) \\
& \leq\left(\frac{1}{3}\right)^{-\frac{N}{2}} \cdot \exp \left(\frac{\rho}{9} \mathbf{h}^{H} \hat{\boldsymbol{S}}\left(\mathbf{E}_{i}^{H} \mathbf{E}_{j} \mathbf{E}_{j}^{H} \mathbf{E}_{i}-\mathbf{I}_{R L}\right) \hat{\boldsymbol{S}} \mathbf{h}\right) .
\end{aligned}
$$

Taking expectation over $\mathbf{h}$ on both sides and using the MGF formula in [26] again, it follows that

$$
\begin{aligned}
\mathrm{P} & \left(w>0 \mid \mathbf{C}_{i}, \hat{\boldsymbol{S}}\right) \\
& \leq\left(\frac{1}{3}\right)^{-\frac{N}{2}} \operatorname{det}^{-1}\left(\mathbf{I}_{R L}+\frac{\rho}{9} \hat{\boldsymbol{S}} \boldsymbol{\Sigma}^{2}\left(\mathbf{I}_{R L}-\mathbf{E}_{i}^{H} \mathbf{E}_{j} \mathbf{E}_{j}^{H} \mathbf{E}_{i}\right)\right) \\
& \leq\left(\frac{1}{3}\right)^{-\frac{N}{2}}\left(\frac{\rho}{9}\right)^{-d_{i, j}} \prod_{k=1}^{d_{i, j}} \lambda_{k}^{-1}\left(\hat{\boldsymbol{S}} \boldsymbol{\Sigma}^{2} \mathbf{Q}\right),
\end{aligned}
$$

where $\mathbf{Q}=\mathbf{I}_{R L}-\mathbf{E}_{j}^{H} \mathbf{E}_{i} \mathbf{E}_{i}^{H} \mathbf{E}_{j}$ as defined in (7), and $d_{i, j}$ is the rank of $\hat{\boldsymbol{S}} \boldsymbol{\Sigma}^{2} \mathrm{Q}$. In (18), the decay rate of the conditional PEP scales with $n_{\mathbf{S}_{i}} L$ when $\mathbf{Q}$ is full rank, similar to (7).

3) Average Pairwise Error Probability: Following a similar argument as in Section III-A3, we can obtain

$$
\mathrm{P}\left(\mathbf{C}_{i} \rightarrow \mathbf{C}_{j} \mid \mathbf{C}_{i}\right) \doteq \rho^{-R \min \left\{L_{s}, L\right\}} .
$$

With the result of Proposition 1 and (19) we have

$$
\begin{aligned}
& \rho^{-R \min \left\{L_{s}, L\right\}} \\
& \quad \doteq \mathrm{PEP}_{\mathrm{MLA}} \leq \mathrm{PEP}_{\mathrm{MLB}} \leq \mathrm{PEP}_{\text {subopt }} \doteq \rho^{-R \min \left\{L_{s}, L\right\}},
\end{aligned}
$$

where $\mathrm{PEP}_{\mathrm{MLA}}, \mathrm{PEP}_{\mathrm{MLB}}$, and $\mathrm{PEP}_{\text {subopt }}$ represent the PEP under the decision rule in (5), (11), and (12), respectively. In (20), the first diversity equivalence has been developed in Section III-A. The first inequality follows from the fact that the MLA decoder described in Section III-A has additional information, i.e., the relay decoding status, the second inequality follows directly from the optimality of the MLB decoder as compared to the suboptimal decoder, and the third inequality comes from (19). By the squeeze theorem we conclude that

$$
\mathrm{PEP}_{\mathrm{MLA}} \doteq \mathrm{PEP}_{\mathrm{MLB}} \doteq \mathrm{PEP}_{\text {subopt }} .
$$

The first diversity equivalence indicates that the maximum achievable diversity is the same $R \min \left\{L_{s}, L\right\}$ with or without knowledge of the channel statistics and the relay status at the destination. That is, the destination can still retrieve full diversity even without knowledge of the channel statistics and the relay decoding status. The second diversity equivalence indicates that both the optimal ML decoding and the suboptimal correlator-based decoding yield the same maximum achievable diversity for a completely noncoherent receiver.

Remark 1: As seen in (7) and (18), the decaying rate of the error probability scales with $\rho^{-R L}$ when $\mathbf{Q}$ is full rank for an arbitrary pair of distinct pseudo-codeword matrices $\mathbf{E}_{i}$ and $\mathbf{E}_{j}$. This full-rank requirement is analogous to the requirement in noncoherent SFC codeword construction for conventional MIMO-OFDM [19]. This suggests that the codeword matrices constructed based on the coding criteria in [19] can be adopted here to achieve full diversity of the system. 


\section{DiVersity Analysis With IMPERFECT RELAY CENSORING}

In this section, we analyze the maximum achievable diversity of the noncoherent system with imperfect relay censoring. We present interesting new results of how error probabilities $p_{s}^{r}$, $p_{1 \mid 0}^{r}$, and $p_{0 \mid 1}^{r}$ affect the system's achievable diversity at the destination node when the destination does not have the relay censoring status information.

Without loss of generality, we assume that the transmitted message $\mathbf{s}$ from the source in Slot I is $\mathbf{s}_{i}$. The noncoherent ML decoding at the destination with no information of the relay status is

$$
\begin{aligned}
\hat{\boldsymbol{C}}_{\mathrm{ML}} & =\underset{\mathbf{C}_{i} \in \mathcal{C}}{\arg \max } p\left(\mathbf{r}_{d} \mid \mathbf{C}_{i}\right) \\
& =\underset{\mathbf{C}_{i} \in \mathcal{C}}{\arg \max } E_{\hat{\boldsymbol{S}}_{0}^{K-1}}\left[p\left(\mathbf{r}_{d} \mid \mathbf{C}_{i}, \hat{\boldsymbol{S}}_{0}^{K-1}\right)\right],
\end{aligned}
$$

where $\mathbf{r}_{d}$ is given in (3) with the possible existence of harmful relays due to imperfect censoring, and $\hat{\boldsymbol{S}}_{0}^{K-1} \triangleq\left(\hat{\boldsymbol{S}}_{0}, \hat{\boldsymbol{S}}_{1}, \ldots\right.$, $\left.\hat{\boldsymbol{S}}_{K-1}\right)$ collects the relay status with respect to all possible transmitted signals. We first obtain the conditional probability mass function (PMF) of $\hat{\boldsymbol{S}}_{0}^{K-1}$ in the following.

Lemma 2: Suppose that the following condition holds: $\mathrm{P}\left(\mathrm{R}_{j}\right.$ active, $\left.\hat{\mathbf{s}}^{j}=\mathbf{s}_{p} \mid \mathbf{s}=\mathbf{s}_{i}\right) \doteq \mathrm{P}\left(\mathrm{R}_{j}\right.$ active, $\left.\hat{\mathbf{s}}^{j}=\mathbf{s}_{q} \mid \mathbf{s}=\mathbf{s}_{i}\right)$ for all relays $\mathrm{R}_{j}, j=1, \ldots, R$ and any $p \neq q \neq i$. Then, the conditional PMF $\mathrm{P}\left(\hat{\boldsymbol{S}}_{0}^{K-1} \mid \mathbf{C}_{i}\right)$ is

$$
\begin{aligned}
\mathrm{P}\left(\hat{\boldsymbol{S}}_{0}^{K-1} \mid \mathbf{C}_{i}\right) \doteq & \prod_{j \in \mathcal{R}_{u \mid i}}\left(1-p_{s}^{j}\right)\left(1-p_{1 \mid 0}^{j}\right) \\
& \times \prod_{k \in \mathcal{R}_{s \mid i}}\left(\left(1-p_{s}^{k}\right) p_{1 \mid 0}+\left(1-p_{0 \mid 1}^{k}\right) p_{s}^{k}\right) \\
& \times \prod_{l \in \mathcal{R}_{h \mid i}} p_{0 \mid 1}^{l} p_{s}^{l},
\end{aligned}
$$

where $\mathcal{R}_{u \mid i} \triangleq\left\{r: S_{i, r}=1 \mid \mathbf{s}=\mathbf{s}_{i}\right\}$ is the set of useful relays, $\mathcal{R}_{s \mid i} \triangleq\left\{r: S_{m, r}=0, \forall m \mid \mathbf{s}=\mathbf{s}_{i}\right\}$ is the set of silent relays, and $\mathcal{R}_{h \mid i} \triangleq\left\{r: S_{m, r}=1, m \neq i \mid \mathbf{s}=\mathbf{s}_{i}\right\}$ is the set of harmful relays, when the transmitted message in Slot I is $\mathbf{s}_{i}$.

Proof: See Appendix A.

Note that the condition $\mathrm{P}\left(\mathrm{R}_{j}\right.$ active, $\left.\hat{\mathrm{s}}^{j}=\mathbf{s}_{p} \mid \mathbf{s}=\mathbf{s}_{i}\right) \doteq$ $\mathrm{P}\left(\mathrm{R}_{j}\right.$ active, $\left.\hat{\mathbf{s}}^{j}=\mathbf{s}_{q} \mid \mathbf{s}=\mathbf{s}_{i}\right)$ in Lemma 2 states that the probabilities that $R_{j}$ erroneously decodes as $\mathbf{s}_{p}$ and as $\mathbf{s}_{q}$ decay at the same rate. This condition holds when the distances from $\mathbf{s}_{i}$ to $\mathbf{s}_{p}$ and to $\mathbf{s}_{q}$ follow $\left\|\mathbf{s}_{i}-\mathbf{s}_{p}\right\|=\eta \cdot\left\|\mathbf{s}_{i}-\mathbf{s}_{q}\right\|$, where $\eta$ is irrelevant to $\rho$. Most modulation schemes satisfy this distance relation.

To facilitate further mathematical development without loss of analytical insights, we hereafter consider a common $p_{s}^{r}, p_{1 \mid 0}^{r}$, and $p_{0 \mid 1}^{r}$ for all relays (thus, the superscript $r$ will be dropped). Then, (22) simplifies to

$$
\begin{aligned}
\mathrm{P}\left(\hat{\boldsymbol{S}}_{0}^{K-1} \mid \mathbf{C}_{i}\right) \doteq & \left(\left(1-p_{s}\right)\left(1-p_{1 \mid 0}\right)\right)^{n_{\mathbf{S}_{i}}} \times \prod_{m \neq i}\left(p_{0 \mid 1} p_{s}\right)^{n_{\mathbf{S}_{m}}} \\
& \times\left(\left(1-p_{s}\right) p_{1 \mid 0}+p_{s}\left(1-p_{0 \mid 1}\right)\right)^{n_{0}}, \quad(23)
\end{aligned}
$$

where $n_{\mathbf{S}_{m}} \triangleq \sum_{r=1}^{R} S_{m, r}$ is the number of active relays that have decoded $\mathbf{s}_{m}(m \neq i)$, and $n_{0} \triangleq R-\sum_{m=0}^{K-1} n_{\mathbf{S}_{m}}$ is the number of silent relays.

For a noncoherent decoding rule without knowledge of the relay status (i.e., the noncoherent ML decoder in (21) or the correlator-based decoder in (12)), the PEP can be expressed as

$$
\begin{aligned}
\mathrm{P}\left(\mathbf{C}_{i}\right. & \left.\rightarrow \mathbf{C}_{j} \mid \mathbf{C}_{i}\right) \\
& =\sum_{\hat{\boldsymbol{S}}_{0}^{K-1}} \mathrm{P}\left(\hat{\boldsymbol{S}}_{0}^{K-1} \mid \mathbf{C}_{i}\right) \mathrm{P}\left(\mathbf{C}_{i} \rightarrow \mathbf{C}_{j} \mid \mathbf{C}_{i}, \hat{\boldsymbol{S}}_{0}^{K-1}\right) .
\end{aligned}
$$

Note that, from Lemma 1, we only need to consider the dominant term in (24) to analyze the achievable diversity order. To characterize the scaling law of the PEP, we first examine the scaling behavior of the conditional $\operatorname{PMF} \mathrm{P}\left(\hat{\mathbf{S}}_{0}^{K-1} \mid \mathbf{C}_{i}\right)$ as the SNR goes to infinity.

Lemma 3: In high SNR regimes, $\mathrm{P}\left(\hat{\boldsymbol{S}}_{0}^{K-1} \mid \mathbf{C}_{i}\right)$ in (23) is diversity-equivalent to

$$
\mathrm{P}\left(\hat{\boldsymbol{S}}_{0}^{K-1} \mid \mathbf{C}_{i}\right) \doteq\left(p_{0 \mid 1} p_{s}\right)^{\sum_{m \neq i} n_{\mathbf{S}_{m}}}\left(p_{1 \mid 0}+p_{s}\right)^{n_{0}} .
$$

\section{Proof: See Appendix B.}

As suggested by (25), the achievable diversity order depends on the error probabilities $p_{s}, p_{1 \mid 0}$, and $p_{0 \mid 1}$. In what follows, we specifically characterize this relationship in three propositions.

Proposition 2: If $p_{1 \mid 0}$ is a positive constant that does not scale with $\rho$, the diversity order is zero with the noncoherent ML decoder.

Proof: We aim to show that

$$
\mathrm{P}\left(\mathbf{C}_{i} \rightarrow \mathbf{C}_{j} \mid \mathbf{C}_{i}\right) \doteq \zeta_{1}
$$

for some constant $\zeta_{1}$ irrelevant to $\rho$. To see this, consider the dominant summation term in (24) where $\hat{\boldsymbol{S}}_{0}^{K-1}=$ $\left(\mathbf{0}_{R L}, \mathbf{0}_{R L}, \ldots, \mathbf{0}_{R L}\right) \triangleq \mathbf{O}$. This leads to $\hat{\boldsymbol{S}}_{0}=\mathbf{I}_{R L}$ and $n_{0}=$ $R$, i.e., no relay helps forward the information from the source to the destination and the destination node receives only noise in Slot II. Thus, we have $\mathrm{P}\left(\mathbf{C}_{i} \rightarrow \mathbf{C}_{j} \mid \mathbf{C}_{i}, \hat{\boldsymbol{S}}_{0}^{K-1}=\mathbf{O}\right) \doteq \zeta_{2}$ for some constant $\zeta_{2}$. This fact, coupled with (25) in Lemma 3 , leads to

$$
\begin{aligned}
\mathrm{P}\left(\hat{\boldsymbol{S}}_{0}^{K-1}=\mathbf{O} \mid \mathbf{C}_{i}\right) \mathrm{P} & \left(\mathbf{C}_{i} \rightarrow \mathbf{C}_{j} \mid \mathbf{C}_{i}, \hat{\boldsymbol{S}}_{0}^{K-1}=\mathbf{O}\right) \\
& \doteq\left(p_{1 \mid 0}+p_{s}\right)^{R} \zeta_{2} \doteq\left(p_{1 \mid 0}\right)^{R} \zeta_{2}
\end{aligned}
$$

where the second diversity equivalence follows from the fact that $p_{s} \doteq \rho^{-L_{s}}$ and that $p_{1 \mid 0}$ is a constant. As the diversity is determined by the dominant term in (24), we reach (26).

Proposition 2 demonstrates that, if $p_{1 \mid 0}$ does not scale with $\rho$, the decoding error will be dominated by the event that all relays are useless relays in high SNR regimes.

Proposition 3: If $p_{0 \mid 1}$ is a positive constant that does not scale with $\rho$, the maximum achievable diversity order is upper bounded by $\left\lceil\frac{R}{2}\right\rceil L_{s}$ with the noncoherent ML decoder.

Proof: Since the diversity is determined by the dominant term in (24) as shown in Lemma 1, it suffices to examine whether there exists one term in (24) that has 
the diversity $\left\lceil\frac{R}{2}\right\rceil L_{s}$ for the upper bound. We consider the case that $\hat{\boldsymbol{S}}_{i}=\mathbf{I}_{L} \otimes \operatorname{diag}\{\overbrace{1, \ldots, 1}^{\left\lfloor\frac{R}{2}\right\rfloor 1^{\prime} s}, 0, \ldots, 0\}, \hat{\boldsymbol{S}}_{j}=\mathbf{I}_{L} \otimes$ $\operatorname{diag}\{0, \ldots, 0, \overbrace{1, \ldots, 1}^{\left\lfloor\frac{R}{2}\right\rfloor 1^{\prime} s}\}$, and $\hat{\boldsymbol{S}}_{m}=\mathbf{0}_{R L}$ for $m \neq i, j$. For notational brevity, we denote the relay status collectively as $\hat{\boldsymbol{S}}_{0}^{K-1}=\mathbf{V}_{i j}$. Since the decision at the destination has no preference for $\mathbf{C}_{i}$ over $\mathbf{C}_{j}$, we have $\mathrm{P}\left(\mathbf{C}_{i} \rightarrow \mathbf{C}_{j} \mid \mathbf{C}_{i}, \hat{\boldsymbol{S}}_{0}^{K-1}=\right.$ $\left.\mathbf{V}_{i j}\right)=\frac{1}{2}$. Using (25) we can obtain

$$
\begin{aligned}
\mathrm{P}\left(\hat{\boldsymbol{S}}_{0}^{K-1}=\mathbf{V}_{i j} \mid\right. & \left.\mathbf{C}_{i}\right) \mathrm{P}\left(\mathbf{C}_{i} \rightarrow \mathbf{C}_{j} \mid \mathbf{C}_{i}, \hat{\boldsymbol{S}}_{0}^{K-1}=\mathbf{V}_{i j}\right) \\
& \doteq \frac{1}{2}\left(p_{0 \mid 1} p_{s}\right)^{\left\lfloor\frac{R}{2}\right\rfloor}\left(p_{1 \mid 0}+p_{s}\right)^{R-2\left\lfloor\frac{R}{2}\right\rfloor} \\
& \geq \frac{1}{2}\left(p_{0 \mid 1} p_{s}\right)^{\left\lfloor\frac{R}{2}\right\rfloor}\left(p_{s}\right)^{R-2\left\lfloor\frac{R}{2}\right\rfloor} \\
& \doteq \rho^{-R L_{s}+\left\lfloor\frac{R}{2}\right\rfloor L_{s}}=\rho^{-\left\lceil\frac{R}{2}\right\rceil L_{s},}
\end{aligned}
$$

where the last diversity equivalence follows from the fact that $p_{s} \doteq \rho^{-L_{s}}$ and $p_{0 \mid 1}$ is a constant.

It is worthwhile to note that by setting $p_{1 \mid 0}=0$ and $L_{s}=1$, Proposition 3 reduces to the special case considered previously [10] for noncoherent wireless DF relaying with binary frequency shift keying (BFSK) modulation over flat fading channels.

Finally, we analyze how the scaling laws of $p_{0 \mid 1}$ and $p_{1 \mid 0}$ affect the maximal achievable diversity order when the receiver employs the noncoherent correlator-based decoder in (12) with imperfect relay censoring.

Lemma 4: The conditional PEP for the correlator-based decoder has the property

$$
\mathrm{P}\left(\mathbf{C}_{i} \rightarrow \mathbf{C}_{j} \mid \mathbf{C}_{i}, \hat{\mathbf{S}}_{0}^{K-1} \in \mathcal{U}_{i j}\right) \doteq \zeta_{3}
$$

where $\mathcal{U}_{i j} \triangleq\left\{\hat{\boldsymbol{S}}_{0}^{K-1}: \hat{\boldsymbol{S}}_{i} \neq \mathbf{0}_{R L}, \hat{\boldsymbol{S}}_{j} \neq \mathbf{0}_{R L}\right.$, and $\hat{\boldsymbol{S}}_{m}=\mathbf{0}_{R L}$ for all $m \neq i, j\}$ represents the relay status where there is at least one useful relay and at least one harmful relay and where the harmful relay(s) all decoded the messages as $\mathbf{s}_{j}$, and $\zeta_{3}$ is a constant irrelevant of $\rho$.

Proof: See Appendix C.

Proposition 4: If $p_{0 \mid 1}$ and $p_{1 \mid 0}$ scale with $\rho$ as $p_{0 \mid 1} \doteq \rho^{-L_{0 \mid 1}}$ and $p_{1 \mid 0} \doteq \rho^{-L_{1 \mid 0}}$, respectively, the maximum achievable diversity order is $\min \left\{L_{s}+L_{0 \mid 1}, R \cdot \min \left\{L_{1 \mid 0}, L\right\}\right\}$ with the correlator-based decoder.

Proof: We first divide all summation terms (i.e., all possible relay status) in (24) into three different classes and then within each class we find the dominant terms which contribute to the diversity order.

Consider the first class that corresponds to $\hat{\boldsymbol{S}}_{0}^{K-1} \in \mathcal{U}_{i j}$, where $\mathcal{U}_{i j}$ is defined in Lemma 4 . For this class, $\sum_{m \neq i} n_{\mathbf{S}_{m}}=$ $n_{\mathbf{S}_{j}} \geq 1$ and $\mathrm{P}\left(\hat{\boldsymbol{S}}_{0}^{K-1} \mid \mathbf{C}_{i}\right) \leq \rho^{-\left(L_{0 \mid 1}+L_{s}\right)}$ from (25). Using the result in Lemma 4 , we can deduce that the dominant term in (24) within the class $\hat{\boldsymbol{S}}_{0}^{K-1} \in \mathcal{U}_{i j}$ has an exponent $L_{0 \mid 1}+L_{s}$, which occurs when $n_{\mathbf{S}_{j}}=1$ and $n_{0}=0$.
TABLE I

\begin{tabular}{|c|c|c|c|}
\hline r & $K$ & Codeword $\mathbf{C}_{i}$ & {$\left[u_{0}, u_{1}, \ldots, u_{15}\right]$} \\
\hline 2 & 8 & $\Phi^{i}\left[\mathbf{f}_{1} \mathbf{f}_{3}\right]$ & {$\left[\begin{array}{llllllllllllllll}1 & 0 & 3 & 4 & 1 & 0 & 3 & 4 & 1 & 0 & 3 & 4 & 1 & 0 & 3 & 4\end{array}\right]$} \\
\hline & 16 & $\boldsymbol{\Phi}^{i}\left[\mathbf{f}_{1} \mathbf{f}_{3}\right]$ & {$\left[\begin{array}{lllllllllll}1 & 4 & 3 & 0 & 1 & 8 & 3 & 12 & 1 & 4\end{array}\right]$} \\
\hline & 8 & $\Phi^{i}\left[\mathbf{f}_{1} \mathbf{f}_{3} \mathbf{f}_{5} \mathbf{f}_{7}\right]$ & {$\left[\begin{array}{llllllllllllllll}1 & 0 & 3 & 4 & 1 & 0 & 3 & 4 & 1 & 0 & 3 & 4 & 1 & 0 & 3 & 4\end{array}\right]$} \\
\hline & 16 & $\Phi^{i}\left[\mathbf{f}_{1} \mathbf{f}_{3} \mathbf{f}_{5} \mathbf{f}_{7}\right]$ & {$\left[\begin{array}{llllllllllllllll}1 & 4 & 3 & 0 & 1 & 8 & 3 & 12 & 1 & 4 & 3 & 0 & 1 & 8 & 3 & 1\end{array}\right]$} \\
\hline
\end{tabular}

The Codebook $\mathcal{C}$ FOR SLOt II TRANSMission, WHERE $\boldsymbol{\Phi}=\operatorname{diag}\left\{e^{j \frac{2 \pi}{K} u_{0}}, \ldots, e^{j \frac{2 \pi}{K} u_{(N-1)}}\right\}$ WiTH $0 \leq u_{k} \leq K-1$ FOR $k=0,1, \ldots, N-1$, AND $\mathbf{f}_{i}$ IS THE $i$ TH COLUMN OF AN $N \times N$ FFT MATRIX

Consider the second class that corresponds to the absence of any harmful relay, i.e., $\sum_{m \neq i} n_{\mathbf{S}_{m}}=0$. For this class, we can directly use the result presented in Section III-B that $\mathrm{P}\left(\mathbf{C}_{i} \rightarrow\right.$ $\left.\mathbf{C}_{j} \mid \mathbf{C}_{i}, \hat{\boldsymbol{S}}_{0}^{K-1}\right) \doteq \rho^{-k_{1} L}$ and $\mathrm{P}\left(\hat{\boldsymbol{S}}_{0}^{K-1} \mid \mathbf{C}_{i}\right) \doteq \rho^{-k_{2} \min \left\{L_{s}, L_{1 \mid 0}\right\}}$, where $k_{1}$ and $k_{2}$ are the number of useful relays and silent relays, respectively, with $k_{1}+k_{2}=R$ in this class.

Consider the third class that corresponds to all other relay status. Since there is at least one harmful relay, we have $\mathrm{P}\left(\hat{\boldsymbol{S}}_{0}^{K-1} \mid \mathbf{C}_{i}\right) \leq \rho^{-\left(L_{0 \mid 1}+L_{s}\right)}$ and $\mathrm{P}\left(\hat{\boldsymbol{S}}_{0}^{K-1} \mid \mathbf{C}_{i}\right) \mathrm{P}\left(\mathbf{C}_{i} \rightarrow\right.$ $\left.\mathbf{C}_{j} \mid \mathbf{C}_{i}, \hat{\boldsymbol{S}}_{0}^{K-1}\right) \leq \rho^{-\left(L_{0 \mid 1}+L_{s}\right)}$ from (25), which implies an exponent in $\rho$ no less than $L_{0 \mid 1}+L_{s}$.

Taking into account all summation terms in (24) and using Lemma 1, we can express the maximum achievable diversity order as

$$
\min \left\{L_{s}+L_{0 \mid 1}, \min _{k_{1}+k_{2}=R}\left(k_{1} L+k_{2} \cdot \min \left\{L_{1 \mid 0}, L_{s}\right\}\right)\right\} \text {. }
$$

Solving the minimization completes the proof.

Proposition 4 suggests that when $L_{s}+L_{0 \mid 1}$ is dominantly small, the system diversity does not scale with the number of relays $R$ when the destination node adopts the correlator-based decoder. Furthermore, different from the result presented in Section III-B with perfect relay censoring, here, with imperfect relay censoring, the suboptimal correlator-based decoder may not attain the diversity of order $R \cdot \min \left\{L_{s}, L\right\}$ when $L_{0 \mid 1}$ or $L_{1 \mid 0}$ is small. Note that the derivation of the system diversity with the noncoherent ML decoder is difficult since there exists no closed-form ML decision rule, as shown in (21), (23), and (24).

\section{Simulation Results}

Here, we present the decoding block error rate (BLER) performance results at the destination. We set $L_{s}=2, L=2$, and $N=16$. The channel gains are complex Gaussian and are independent across different channel paths, node pairs, and symbol blocks (time instants). The SNR is defined as the total transmit power from the $R$ relays per subchannel, i.e., $\mathrm{SNR}=$ $\rho R / N$ with the noise power normalized to one. The transmit power of the source node is $\rho$. The relay decoding error rate is $p_{s} \doteq \rho^{-L_{s}}$. As commented in Remark 1, the codebook used in Slot II transmission is constructed by following a similar procedure as in [19] and is summarized in Table I.

\section{A. Perfect Relay Censoring}

We first present the results for perfect relay censoring, i.e., $p_{1 \mid 0}=0$ and $p_{0 \mid 1}=0$. 


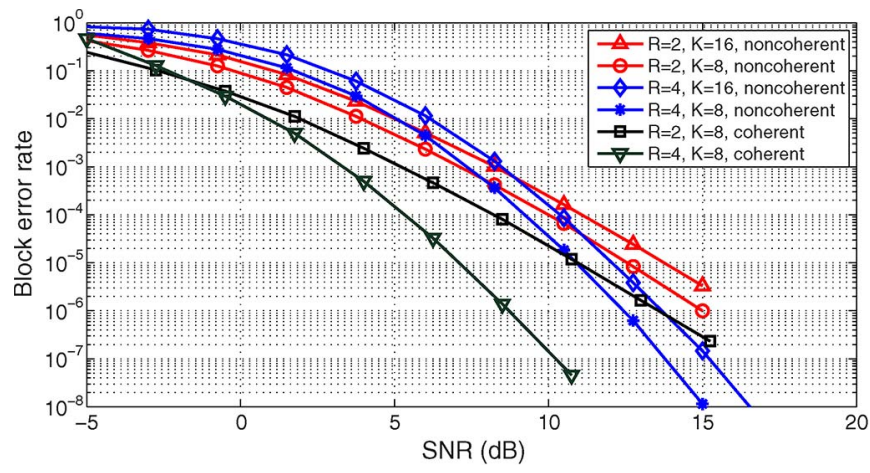

Fig. 2. The BLER performance for the noncoherent partial-knowledge ML decoder in (5) and coherent partial-knowledge ML decoder [28], [29], both with perfect relay censoring, for different configurations of $R$ and $K$.

1) Noncoherent Receiver With Partial Information: The BLER performance results with partial information of channel statistics and relay decoding status at the destination are presented in Fig. 2 for the four combinations of $R=2,4$ (the number of relays) and $K=8,16$ (the codebook size). The channel power delay profile (PDP) is uniform, i.e., $\sigma_{r, 0}^{2}=\sigma_{r, 1}^{2}=$ $0.5, \forall r$ in the simulation. The results show that a diversity of order 4 and 8 is achieved for $R=2$ and $R=4$, respectively, confirming that the potential diversity of distributed relays is identical to that of conventional MIMO-OFDM systems with noncoherent SFC [19]. The difference between the two curves with $R=2$ (and respectively $R=4$ ) corresponds to the coding gain as a result of the different code rates [19].

In Fig. 2, we also show the BLER performance of the coherent receiver with known relay decoding status for $R=2$, $K=8$ and $R=4, K=8$. The same space-frequency code $\mathcal{C}$ presented in Table I is used for encoding in the coherent case, since this codebook $\mathcal{C}$ also satisfies the full diversity condition specified in [28], [29] for coherent space-frequency coded systems. As can be seen, both coherent and noncoherent SFC cooperative relaying systems with the ML decoding achieve full diversity, which confirms that the noncoherent SFC MIMOOFDM system requiring no instantaneous CSI at the receiver can achieve a full diversity as its coherent counterpart [19]. The coherent case, as expected, has a coding gain advantage over the noncoherent case, because instantaneous CSI is known at the coherent receiver while only statistical CSI is available to the noncoherent receiver for the decoding.

Fig. 3 plots the BLER results for different PDPs, with $R=2$. As can be seen, all curves exhibit the same diversity of order 4 . Furthermore, the BLER performance degrades as the channel has a more asymmetric PDP. This can be explained by (7). When the total power $\sum_{l=0}^{L-1} \sigma_{r, l}^{2}$ is a constant of unity, the magnitude of $\lambda^{-1}\left(\hat{\boldsymbol{S}} \boldsymbol{\Sigma}^{2} \mathbf{Q}\right)$, which can be approximated by $\lambda^{-1}\left(\boldsymbol{\Sigma}^{2}\right)=\prod_{r=1}^{R}\left(\sigma_{r, 0}^{2} \sigma_{r, 1}^{2}\right)^{-1}$, increases as the PDP becomes more asymmetric due to the arithmetic-geometric inequality. This agrees with the intuition that, when the PDP is more asymmetric, more power is needed to make the weaker channel contribute to the maximum diversity. As shown in Fig. 3, the diversity order for PDP $\left[\sigma_{r, 0}^{2} \sigma_{r, 1}^{2}\right]=[0.990 .01]$ does not reach 4 until the SNR rises above $20 \mathrm{~dB}$ or so.

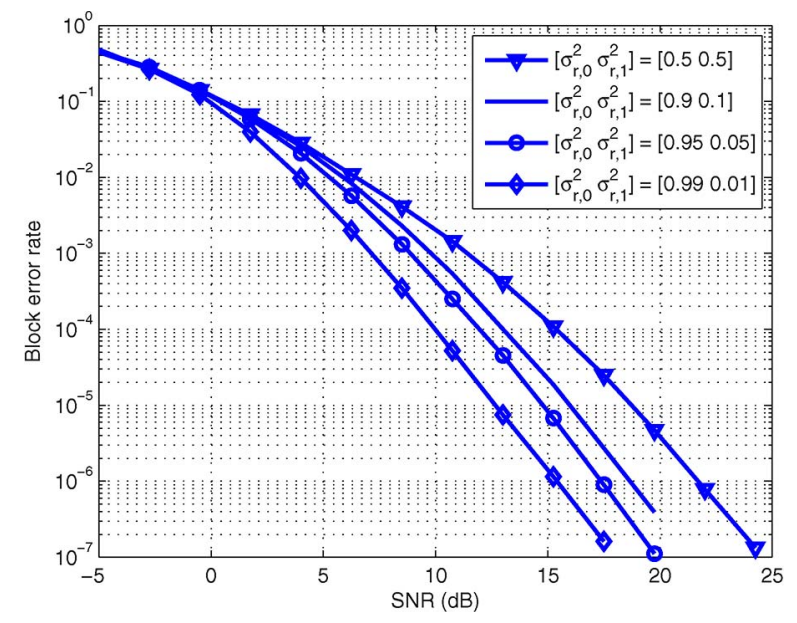

Fig. 3. The BLER performance for the noncoherent partial-knowledge ML decoder in (5) with perfect relay censoring, for different PDPs.
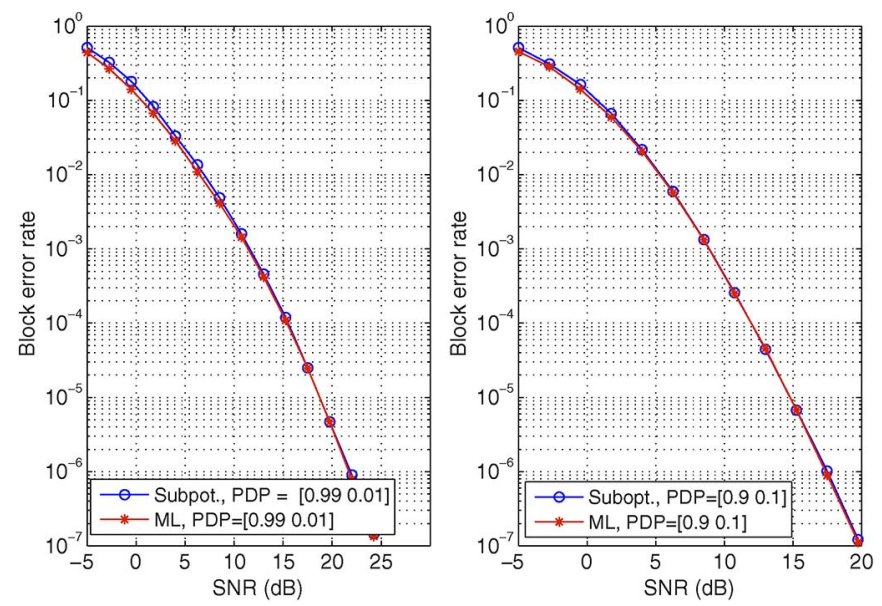

Fig. 4. The BLER performance for the suboptimal correlator-based decoder in (12) and the noncoherent partial-knowledge ML decoder in (5) with perfect relay censoring, for two different PDPs, i.e., $\left[\sigma_{r, 0}^{2} \sigma_{r, 1}^{2}\right]=\left[\begin{array}{lll}0.99 & 0.01\end{array}\right]$ and $\left[\sigma_{r, 0}^{2} \sigma_{r, 1}^{2}\right]=\left[\begin{array}{lll}0.9 & 0.1\end{array}\right]$.

2) Completely Noncoherent Receiver: Fig. 4 shows the BLER performance for the completely noncoherent suboptimal correlator-based decoder in (12) in comparison with the noncoherent partial-knowledge ML decoder in (5), under perfect relay censoring and different channel PDPs. We observe that the two decoders yield almost identical performance even though the completely noncoherent correlator-based decoder does not have partial knowledge. There is a minor performance gap in low SNR regimes, particularly when the PDP is more asymmetric, since correlator-based decoding assumes a uniform PDP. Nevertheless, in high SNR regimes, the performance overlaps. This confirms the near-optimal performance and the diversityachieving capability of a completely noncoherent suboptimal correlator-based decoder under perfect relay censoring.

\section{B. Imperfect Relay Censoring}

Here, we present the results for imperfect relay censoring and examine the effect of $p_{1 \mid 0}$ and $p_{0 \mid 1}=0$ on the BLER 


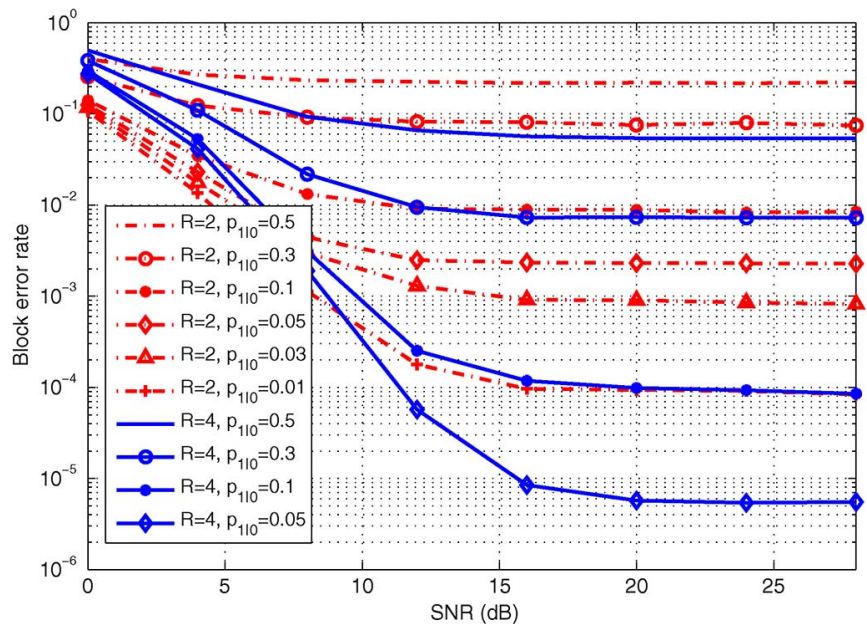

Fig. 5. The BLER performance for the ML decoder in (21) with imperfect relay censoring, for different configurations of $p_{1 \mid 0}$ and $R$, with $p_{0 \mid 1}=0$.

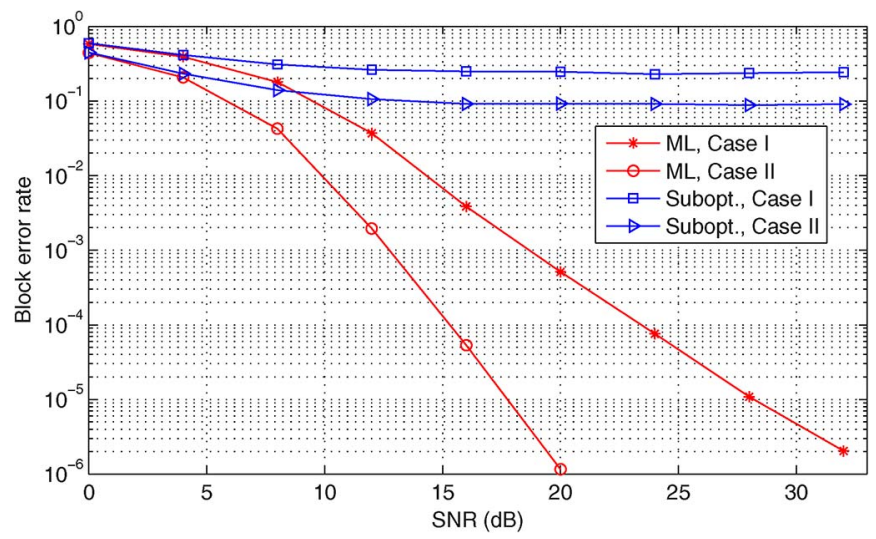

Fig. 6. The BLER performance for the completely noncoherent suboptimal correlator-based decoder and the ML decoder in (21) with imperfect relay censoring, for two cases of the relay decoding status with $R=4$. Case I: 2 useful, 1 useless, and 1 harmful relays. Case II: 3 useful and 1 harmful relays.

performance. Fig. 5 depicts the performance of ML decoding in (21) with $p_{0 \mid 1}=0$ and different settings of $p_{1 \mid 0}$. As can be seen, an error floor occurs for all constant values of $p_{1 \mid 0}$ (even with $p_{0 \mid 1}=0$ ), which justifies Proposition 2. A smaller $p_{1 \mid 0}$ reduces the likelihood that all relays are useless relays and therefore reduces the BLER where the error floor occurs. Also, an increased number of relays reduces the BLER where the error floor occurs, which confirms the analytical result in (27) that the BLER where the error floor occurs approaches $\left(p_{1 \mid 0}\right)^{R}$ as the SNR goes to infinity.

Fig. 6 compares the performance of the suboptimal correlator-based decoder and the optimal ML decoder in (21) for two cases of the relay decoding status with $R=4$, namely, Case I where there are 2 useful, 1 useless, and 1 harmful relays, and Case II where there are 3 useful and 1 harmful relays. The two cases are constructed to illustrate a result of Proposition 4 that the suboptimal correlator-based decoder does not scale with the number of relays if $L_{0 \mid 1}$ is small (i.e., there is a nonnegligible probability of harmful relays). This is verified in Fig. 6, where the correlator-based decoder suffers from an error floor for both cases with one harmful relay. In contrast, the ML decoder does not suffer from an error floor. Also, as

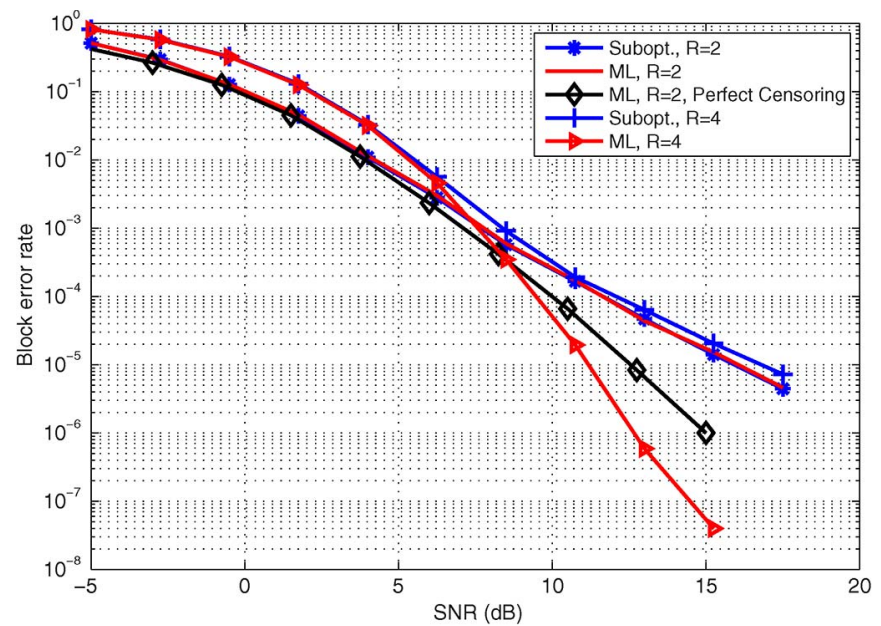

Fig. 7. The BLER performance for the completely noncoherent suboptimal correlator-based decoder and the ML decoder in (21) with imperfect relay censoring $\left(p_{1 \mid 0}=0\right.$ and $\left.p_{0 \mid 1}=1\right)$, as well as the noncoherent partial-knowledge ML decoder with perfect relay censoring.

shown in Fig. 6, Case II outperforms Case I with the same total number of relays, indicating that the relay conditions may affect the diversity order of the system with a completely noncoherent ML decoder.

Fig. 7 shows the performance of the suboptimal correlatorbased decoder and the optimal ML decoder in (21) when all relays are active, i.e., $p_{1 \mid 0}=0$ and $p_{0 \mid 1}=1$. Several observations can be made. First, the diversity order is 2 for the suboptimal decoder for both $R=2$ and $R=4$. This agrees well with the analytical result in Proposition 4 with parameters $L_{s}=2, L=2, p_{0 \mid 1}=1 \doteq \rho^{-0}$, and $p_{1 \mid 0}=0 \doteq \rho^{-\infty}$. In this case, increasing the number of relays does not increase the diversity of the system with correlator-based decoding. Second, the ML decoder achieves a diversity of order 2 and 4 for $R=$ 2 and $R=4$, respectively. This shows that the ML decoder can yield the maximal achievable diversity of order $\left\lceil\frac{R}{2}\right\rceil L_{s}$ as stated in Proposition 3. Third, by comparing the performance for decoders with perfect and imperfect relay censoring for $R=2$, it is seen that the imperfection in censoring decreases the diversity gain.

\section{CONCLUSION}

We have studied the diversity of a noncoherent distributed SFC wireless two-hop relay system with DF relaying. We approached the problem from a previously unexamined perspective that takes into account both perfect and imperfect relay censoring to examine the potential of the system under practical operation. The main findings are summarized as follows:

- Perfect relay censoring: We show that a diversity of order $R \cdot \min \left\{L_{s}, L\right\}$ can be achieved with the optimal ML decoder with or without partial information of channel statistics and relay decoding status at the noncoherent receiver. The same diversity order was shown also achievable with a suboptimal correlator-based decoder, which was proposed as a convenient but motivated scheme for analytical development. Our results suggest that with perfect relay censoring the diversity of the noncoherent distributed SFC 
system is on average identical to that of the conventional SFC MIMO-OFDM system.

- Imperfect relay censoring: We characterize the relationship between censoring error probabilities $p_{1 \mid 0}$ (which corresponds to useless relays) and $p_{0 \mid 1}$ (which corresponds to harmful relays) and the diversity with a completely noncoherent receiver. If $p_{1 \mid 0}$ is a constant that does not scale with the transmit power of the relay $(\rho)$, the diversity order is zero with the ML decoder. If $p_{0 \mid 1}$ is a constant that does not scale with the transmit power of the relay, the diversity order is upper bounded by $\left\lceil\frac{R}{2}\right\rceil L_{s}$ with the ML decoder. If $p_{0 \mid 1}$ and $p_{1 \mid 0}$ scale with $\rho$ as $p_{0 \mid 1} \doteq \rho^{-L_{0 \mid 1}}$ and $p_{1 \mid 0} \doteq \rho^{-L_{1 \mid 0}}$, respectively, the diversity order is given by $\min \left\{L_{s}+L_{0 \mid 1}, R \cdot \min \left\{L_{1 \mid 0}, L\right\}\right\}$ with the correlatorbased decoder. The results suggest that a carefully implemented relay censoring scheme is crucial in achieving the full diversity potential of a distributed relay system.

\section{APPENDIX A}

PROOF OF LEMMA 2

Given transmitted $\mathbf{s}_{i}$ in Slot I, the relays can be grouped into three disjoint sets: 1 ) the set of useful relays $\mathcal{R}_{u \mid i}, 2$ ) the set of silent relays $\mathcal{R}_{s \mid i}$, and 3) the set of harmful relays $\mathcal{R}_{h \mid i}$. The probabilities that a relay is in each set are characterized as follows.

The probability that the $j$ th relay $\mathrm{R}_{j}$ is in $\mathcal{R}_{u \mid i}$ is

$$
\begin{aligned}
\mathrm{P}\left(\mathrm{R}_{j} \in \mathcal{R}_{u \mid i}\right) & =\mathrm{P}\left(S_{i, j}=1 \mid \mathbf{s}=\mathbf{s}_{i}\right) \\
& =\mathrm{P}\left(\mathrm{R}_{j} \text { active, } \hat{\mathbf{s}}^{j}=\mathbf{s}_{i} \mid \mathbf{s}=\mathbf{s}_{i}\right) \\
& =\left(1-p_{s}^{j}\right)\left(1-p_{1 \mid 0}^{j}\right) .
\end{aligned}
$$

The probability that the $k$ th relay $\mathrm{R}_{k}$ is in $\mathcal{R}_{s \mid i}$ is

$$
\begin{aligned}
\mathrm{P}\left(\mathrm{R}_{k} \in \mathcal{R}_{s \mid i}\right)= & \mathrm{P}\left(\mathrm{R}_{k} \text { silent, } \hat{\mathbf{s}}^{k}=\mathbf{s} \mid \mathbf{s}=\mathbf{s}_{i}\right) \\
& +\mathrm{P}\left(\mathrm{R}_{k} \text { silent, } \hat{\mathbf{s}}^{k} \neq \mathbf{s} \mid \mathbf{s}=\mathbf{s}_{i}\right) \\
= & \left(1-p_{s}^{k}\right) p_{1 \mid 0}^{k}+\left(1-p_{0 \mid 1}^{k}\right) p_{s}^{k} .
\end{aligned}
$$

The probability that the $l$ th relay $\mathrm{R}_{l}$ is in $\mathcal{R}_{h \mid i}$ and has erroneously decoded $\mathbf{s}_{i}$ as $\hat{\mathbf{s}}^{l}=\mathbf{s}_{m}(m \neq i)$ is $\mathrm{P}\left(\mathrm{R}_{l} \in \mathcal{R}_{h \mid i}, \hat{\mathbf{s}}^{l}=\right.$ $\left.\mathbf{s}_{m}\right)=\mathrm{P}\left(\mathrm{R}_{l}\right.$ active, $\left.\hat{\mathbf{s}}^{l}=\mathbf{s}_{m} \mid \mathbf{s}=\mathbf{s}_{i}\right)$. Furthermore, we have

$$
\begin{aligned}
& \mathrm{P}\left(\mathrm{R}_{l} \text { active, } \hat{\mathbf{s}}^{l} \neq \mathbf{s}\right) \\
& \quad=\sum_{i=0}^{K-1} \mathrm{P}\left(\mathrm{R}_{l} \text { active, } \hat{\mathbf{s}}^{l} \neq \mathbf{s} \mid \mathbf{s}=\mathbf{s}_{i}\right) \mathrm{P}\left(\mathbf{s}=\mathbf{s}_{i}\right) \\
& \quad \doteq \sum_{i=0}^{K-1} \mathrm{P}\left(\mathrm{R}_{l} \text { active, } \hat{\mathbf{s}}^{l} \neq \mathbf{s} \mid \mathbf{s}=\mathbf{s}_{i}\right) \\
& \quad=\sum_{i=0}^{K-1} \sum_{j \neq i} \mathrm{P}\left(\mathrm{R}_{l} \text { active }, \hat{\mathbf{s}}^{l}=\mathbf{s}_{j} \mid \mathbf{s}=\mathbf{s}_{i}\right) \\
& \doteq \mathrm{P}\left(\mathrm{R}_{l} \text { active, }, \hat{\mathbf{s}}^{l}=\mathbf{s}_{j} \mid \mathbf{s}=\mathbf{s}_{i}\right)
\end{aligned}
$$

where the second diversity equivalence follows from the condition $\mathrm{P}\left(\mathrm{R}_{j}\right.$ active, $\left.\hat{\mathbf{s}}^{j}=\mathbf{s}_{p} \mid \mathbf{s}=\mathbf{s}_{i}\right) \doteq \mathrm{P}\left(\mathrm{R}_{j}\right.$ active, $\hat{\mathbf{s}}^{j}=\mathbf{s}_{q} \mid \mathbf{s}=$ $\left.\mathbf{s}_{i}\right)$. Thus, $\mathrm{P}\left(\mathrm{R}_{l} \in \mathcal{R}_{h \mid i}, \hat{\mathbf{s}}^{l}=\mathbf{s}_{m}\right) \doteq \mathrm{P}\left(\mathrm{R}_{l}\right.$ active, $\left.\hat{\mathbf{s}}^{l} \neq \mathbf{s}\right)=$ $p_{0 \mid 1} p_{s}$.

Combining the results for the three cases completes the proof.

\section{APPEndix B \\ PROOF OF LEMMA 3}

Taking the logarithm of the right-hand-side of (23) yields

$$
\begin{array}{r}
n_{\mathbf{S}_{i}} \log \left(\left(1-p_{s}\right)\left(1-p_{1 \mid 0}\right)\right)+\sum_{m \neq i} n_{\mathbf{S}_{m}} \log \left(p_{0 \mid 1} p_{s}\right) \\
+n_{0} \log \left(\left(1-p_{s}\right) p_{1 \mid 0}+p_{s}\left(1-p_{0 \mid 1}\right)\right) .
\end{array}
$$

We examine the scaling behaviors of the first and the third term in (28). Since $p_{s} \doteq \rho^{-L_{s}},\left(1-p_{s}\right)\left(1-p_{1 \mid 0}\right)$ must be a constant as $\rho \rightarrow \infty$ regardless of the scaling laws of $p_{1 \mid 0}$. For the third term in (28), if $p_{0 \mid 1}$ is a constant that does not scale with $\rho$, we must have $\left(1-p_{s}\right) p_{1 \mid 0}+p_{s}\left(1-p_{0 \mid 1}\right) \doteq p_{1 \mid 0}+\beta \cdot p_{s}$ for some constant $\beta$, which immediately leads to $\left(1-p_{s}\right) p_{1 \mid 0}+$ $p_{s}\left(1-p_{0 \mid 1}\right) \doteq p_{1 \mid 0}+p_{s}$ since multiplying by a constant does not affect the scaling laws. If $p_{0 \mid 1}$ scales with $\rho$ as $p_{0 \mid 1} \doteq$ $\rho^{-L_{0 \mid 1}}$, we see that $p_{0 \mid 1}$ vanishes rapidly in $1-p_{0 \mid 1}$ when $\rho \rightarrow \infty$, which also leads to $\left(1-p_{s}\right) p_{1 \mid 0}+p_{s}\left(1-p_{0 \mid 1}\right) \doteq$ $p_{1 \mid 0}+p_{s}$.

Substituting these results into (23) yields the following and completes the proof:

$$
\begin{array}{r}
\frac{1}{\log \rho} \log \mathrm{P}\left(\hat{\boldsymbol{S}}_{0}^{K-1} \mid \mathbf{C}_{i}\right) \\
\rightarrow \frac{1}{\log \rho}\left(\sum_{m \neq i} n_{\mathbf{S}_{m}} \log \left(p_{0 \mid 1} p_{s}\right)+n_{0} \log \left(p_{1 \mid 0}+p_{s}\right)\right) \\
\text { as } \rho \rightarrow \infty .
\end{array}
$$

APPENDIX C

PROOF OF LEMMA 4

The received signal at the destination when $\hat{\boldsymbol{S}}_{0}^{K-1} \in \mathcal{U}_{i j}$ is given by

$$
\mathbf{r}_{d}=\sqrt{\rho} \mathbf{E}_{i} \hat{\boldsymbol{S}}_{i} \mathbf{h}+\sqrt{\rho} \mathbf{E}_{j} \hat{\boldsymbol{S}}_{j} \mathbf{h}+\mathbf{n} .
$$

Then, the conditional PEP is

$$
\begin{aligned}
\mathrm{P}\left(\mathbf{C}_{i}\right. & \left.\rightarrow \mathbf{C}_{j} \mid \mathbf{C}_{i}, \hat{\boldsymbol{S}}_{0}^{K-1} \in \mathcal{U}_{i j}\right) \\
& =\mathrm{P}\left(\mathbf{r}_{d}^{H} \mathbf{E}_{j} \mathbf{E}_{j}^{H} \mathbf{r}_{d}-\mathbf{r}_{d}^{H} \mathbf{E}_{i} \mathbf{E}_{i}^{H} \mathbf{r}_{d}>0 \mid \mathbf{C}_{i}, \mathcal{U}_{i j}\right) \\
& =\mathrm{P}\left(\frac{1}{\rho}\left(\mathbf{r}_{d}^{H} \mathbf{E}_{j} \mathbf{E}_{j}^{H} \mathbf{r}_{d}-\mathbf{r}_{d}^{H} \mathbf{E}_{i} \mathbf{E}_{i}^{H} \mathbf{r}_{d}\right)>0 \mid \mathbf{C}_{i}, \mathcal{U}_{i j}\right) \\
& \triangleq \mathrm{P}\left(w_{\rho}>0 \mid \mathbf{C}_{i}, \mathcal{U}_{i j}\right)
\end{aligned}
$$


where $\quad w_{\rho} \triangleq \overline{\mathbf{r}}_{d}^{H} \mathbf{E}_{j} \mathbf{E}_{j}^{H} \overline{\mathbf{r}}_{d}-\overline{\mathbf{r}}_{d}^{H} \mathbf{E}_{i} \mathbf{E}_{i}^{H} \overline{\mathbf{r}}_{d} \quad$ with $\quad \overline{\mathbf{r}}_{d}=\frac{\mathbf{r}_{d}}{\sqrt{\rho}}=$ $\mathbf{E}_{i} \hat{\boldsymbol{S}}_{i} \mathbf{h}+\mathbf{E}_{j} \hat{\boldsymbol{S}}_{j} \mathbf{h}+\frac{\mathbf{n}}{\sqrt{\rho}}$. Note that showing the lemma is equivalent to showing $\lim _{\rho \rightarrow \infty} \mathrm{P}\left(w_{\rho}>0 \mid \mathbf{C}_{i}, \mathcal{U}_{i j}\right)>0$. Define $\quad w_{\infty} \triangleq \tilde{\mathbf{r}}_{d}^{H} \mathbf{E}_{j} \mathbf{E}_{j}^{H} \tilde{\mathbf{r}}_{d}-\tilde{\mathbf{r}}_{d}^{H} \mathbf{E}_{i} \mathbf{E}_{i}^{H} \tilde{\mathbf{r}}_{d}, \quad$ where $\quad \tilde{\mathbf{r}}_{d}=$ $\mathbf{E}_{i} \hat{\boldsymbol{S}}_{i} \mathbf{h}+\mathbf{E}_{j} \hat{\boldsymbol{S}}_{j} \mathbf{h}$. Clearly, $w_{\rho} \rightarrow w_{\infty}$ almost surely as $\rho \rightarrow \infty$. Thus, we only need to show $\mathrm{P}\left(w_{\infty}>0 \mid \mathbf{C}_{i}, \mathcal{U}_{i j}\right)>0$, since convergence almost surely implies convergence in distribution. After simple manipulations we have

$$
\begin{aligned}
w_{\infty}=\mathbf{h}^{H} \hat{\boldsymbol{S}}_{j}(\mathbf{I}- & \left.\mathbf{E}_{j}^{H} \mathbf{E}_{i} \mathbf{E}_{i}^{H} \mathbf{E}_{j}\right) \hat{\boldsymbol{S}}_{j} \mathbf{h} \\
& -\mathbf{h}^{H} \hat{\boldsymbol{S}}_{i}\left(\mathbf{I}-\mathbf{E}_{i}^{H} \mathbf{E}_{j} \mathbf{E}_{j}^{H} \mathbf{E}_{i}\right) \hat{\boldsymbol{S}}_{i} \mathbf{h} \triangleq X-Y,
\end{aligned}
$$

where $X \triangleq \mathbf{h}^{H} \hat{\boldsymbol{S}}_{j}\left(\mathbf{I}_{R L}-\mathbf{E}_{j}^{H} \mathbf{E}_{i} \mathbf{E}_{i}^{H} \mathbf{E}_{j}\right) \hat{\boldsymbol{S}}_{j} \mathbf{h}$ and $Y \triangleq \mathbf{h}^{H} \hat{\boldsymbol{S}}_{i}$ $\left(\mathbf{I}_{R L}-\mathbf{E}_{i}^{H} \mathbf{E}_{j} \mathbf{E}_{j}^{H} \mathbf{E}_{i}\right) \hat{\boldsymbol{S}}_{i} \mathbf{h}$. Since $\mathbf{I}_{R L}-\mathbf{E}_{j}^{H} \mathbf{E}_{i} \mathbf{E}_{i}^{H} \mathbf{E}_{j}$ is positive definite provided that $\mathbf{E}_{i} \neq \mathbf{E}_{j}$, and $X$ and $Y$ are independent generalized chi-square random variables with support $[0, \infty)$, we have

$$
\begin{aligned}
\mathrm{P}\left(w_{\infty}>0 \mid \mathbf{C}_{i}, \mathcal{U}_{i j}\right) & =\mathrm{P}\left(X-Y>0 \mid \mathbf{C}_{i}, \mathcal{U}_{i j}\right) \\
& \geq \mathrm{P}\left(\{X>1\} \cap\{Y<1\} \mid \mathbf{C}_{i}, \mathcal{U}_{i j}\right) \\
& =\mathrm{P}\left(X>1 \mid \mathbf{C}_{i}, \mathcal{U}_{i j}\right) \mathrm{P}\left(Y<1 \mid \mathbf{C}_{i}, \mathcal{U}_{i j}\right) \\
& >0 .
\end{aligned}
$$

This completes the proof.

\section{REFERENCES}

[1] A. Sendonaris, E. Erkip, and B. Aazhang, "User cooperation diversityPart I: System description," IEEE Trans. Commun., vol. 51, no. 11, pp. 1927-1938, Nov. 2003.

[2] J. Laneman, D. Tse, and G. Wornell, "Cooperative diversity in wireless networks: Efficient protocols and outage behavior," IEEE Trans. Inf. Theory, vol. 50, no. 12, pp. 3062-3080, Dec. 2004.

[3] V. Tarokh, N. Seshadri, and A. R. Calderbank, "Space-time codes for high data rate wireless communication: Performance criterion and code construction," IEEE Trans. Inf. Theory, vol. 44, no. 2, pp. 744-765, Mar. 1998

[4] J. Laneman and G. Wornell, "Distributed space-time-coded protocols for exploiting cooperative diversity in wireless network," IEEE Trans. Inf. Theory, vol. 49, no. 10, pp. 2415-2425, Oct. 2003.

[5] Y. Jing and B. Hassibi, "Distributed space-time coding in wireless relay networks," IEEE Trans. Wireless Commun., vol. 5, no. 12, pp. 3524-3536, Dec. 2006.

[6] B. Sirkeci-Mergen and A. Scaglione, "Randomized space-time coding for distributed cooperative communication," IEEE Trans. Signal Process., vol. 55, no. 10, pp. 5003-5017, Oct. 2007.

[7] Y. Li, W. Zhang, and X. Xia, "Distributive high-rate space-frequency codes achieving full cooperative and multipath diversities for asynchronous cooperative communications," IEEE Trans. Veh. Technol., vol. 58, no. 1, pp. 207-217, Jan. 2009.

[8] K. G. Seddik and K. J. R. Liu, "Distributed space-frequency coding over broadband relay channels," IEEE Trans. Wireless Commun., vol. 7, no. 11, pp. 4747-4759, Nov. 2008.

[9] J. Boyer, D. D. Falconer, and H. Yanikomeroglu, "Multihop diversity in wireless relaying channels," IEEE Trans. Commun., vol. 52, no. 10, pp. $1820-1830$, Oct. 2004.

[10] D. Chen and J. N. Laneman, "Modulation and demodulation for cooperative diversity in wireless systems," IEEE Trans. Wireless Commun., vol. 5, no. 7, pp. 1785-1794, Jul. 2006.

[11] F. A. Onat et al., "Threshold selection for SNR-based selective digital relaying in cooperative wireless networks," IEEE Trans. Wireless Commun., vol. 7, no. 11, pp. 4226-4237, Nov. 2008.

[12] F. A. Onat, Y. Fan, H. Yanikomeroglu, and J. S. Thompson, "Asymptotic BER analysis of threshold digital relaying schemes in cooperative wireless systems," IEEE Trans. Wireless Commun., vol. 7, no. 12, pp. 4938-4947, Dec. 2008 .

[13] T. Wang, A. Cano, G. B. Giannakis, and J. N. Laneman, "Highperformance cooperative demodulation with decode-and-forward relays," IEEE Trans. Commun., vol. 55, no. 7, pp. 1427-1438, Jul. 2007.

[14] T. Wang, G. B. Giannakis, and R. Wang, "Smart regenerative relays for link-adaptive cooperative communications," IEEE Trans. Commun., vol. 56, no. 11, pp. 1950-1960, Nov. 2008.

[15] T. Hunter and A. Nosratinia, "Diversity through coded cooperation," IEEE Trans. Wireless Commun., vol. 5, no. 2, pp. 283-289, Feb. 2006.

[16] G. Al-Habian, A. Ghrayeb, M. Hasna, and A. Abu-Dayya, "Thresholdbased relaying in coded cooperative networks," IEEE Trans. Veh. Technol., vol. 60, no. 1, pp. 123-135, Jan. 2011.

[17] B. M. Hochwald and T. L. Marzetta, "Unitary space-time modulation for multiple-antenna communications in Rayleigh flat fading," IEEE Trans. Inf. Theory, vol. 46, no. 2, pp. 543-564, Mar. 2000.

[18] V. Tarokh and I.-M. Kim, "Existence and construction of noncoherent unitary space-time codes," IEEE Trans. Inf. Theory, vol. 48, no. 12, pp. 3112-3117, Dec. 2002.

[19] M. Borgmann and H. Bolcskei, "Noncoherent space-frequency coded MIMO-OFDM," IEEE J. Sel. Areas Commun., vol. 23, no. 9, pp. 17991810, Sep. 2005

[20] M. R. Souryal, "Non-coherent amplify-and-forward generalized likelihood ratio test receiver," IEEE Trans. Wireless Commun., vol. 9, no. 7, pp. 2320-2327, Jul. 2010.

[21] P. Lin, S. Gazor, I.-M. Kim, and D. I. Kim, "Noncoherent amplify-andforward cooperative networks: Robust detection and performance analysis," IEEE Trans. Commun., vol. 61, no. 9, pp. 3644-3659, Sep. 2013.

[22] G. Farhadi and N. C. Beaulieu, "A low complexity receiver for noncoherent amplify-and-forward cooperative systems," IEEE Trans. Commun., vol. 58, no. 9, pp. 2499-2504, Sep. 2010.

[23] T.-H. Chang, W.-K. Ma, C.-Y. Huang, and C.-Y. Chi, "Noncoherent OSTBC-OFDM for MIMO and cooperative communications: Perfect channel identifiability and achievable diversity order," IEEE Trans. Signal Process., vol. 60, no. 9, pp. 4849-4863, Sep. 2012.

[24] M. C. Valenti, D. Torrieri, and T. Ferrett, "Noncoherent physical-layer network coding with FSK modulation: Relay receiver deign issues," IEEE Trans. Commun., vol. 59, no. 9, pp. 2595-2604, Sep. 2011.

[25] P. Herhold, E. Zimmermann, and G. Fettweis, "A simple cooperative extension to wireless relaying," in Proc. Int. Zurich Seminar Commun., Mar. 2004, pp. 36-39.

[26] G. L. Turin, "The characteristic function of Hermitian quadratic forms in complex normal variables," Biometrika, vol. 47, no. 1/2, pp. 199-201, Jun. 1960.

[27] R. A. Horn and C. R. Johnson, Matrix Analysis. New York, NY, USA: Cambridge Univ. Press, 1985.

[28] H. Bolcskei and A. J. Paulraj, "Space-frequency coded broadband OFDM systems," in Proc. IEEE WCNC, 2000, pp. 1-6.

[29] H. Bolcskei and A. J. Paulraj, "Space-frequency codes for broadband fading channels," in Proc. IEEE ISIT, 2001, pp. 219-219.

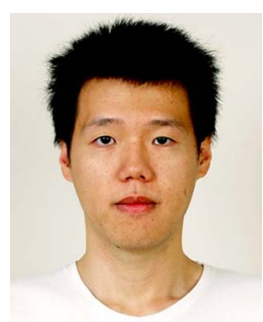

communication systems.
Sung-En Chiu received the B.S. and M.S. degrees in electronics engineering from National Chiao Tung University, Hsinchu, Taiwan, in 2008 and 2010, respectively. He is currently working toward the Ph.D. degree in electrical and computer engineering at the University of California, San Diego, CA, USA. From 2011 to 2013, he served as a 3GPP RAN1 delegate for the Industrial Technology Research Institute, Hsinchu, Taiwan. His current research interests include statistical signal processing, dynamic resource allocation, large-scale MIMO, and next generation 


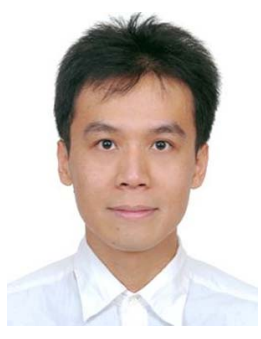

Feng-Tsun Chien (S'02-M'05) received the B.S. degree from National Tsing Hua University, Hsinchu, Taiwan, in 1995, the M.S. degree from National Taiwan University, Taipei, Taiwan, in 1997, and the Ph.D. degree from the University of Southern California, Los Angeles, CA, USA, in 2004, all in electrical engineering. He joined the Department of Electronics Engineering, National Chiao Tung University, Hsinchu, in July 2005 as an Assistant Professor. His current research interests include wireless communications, statistical signal processing, game theoretic resource allocation, and network information theory. He has been serving as a Technical Program Committee Member in ICC (2009-2015), GLOBECOM (2009-2014), WCNC (2010-2015), and PIMRC (2011-2014) and is currently the treasurer of the IEEE Vehicular Technology Society, Taipei Chapter.

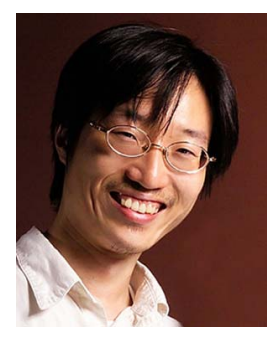

Ronald Y. Chang (M'12) received the B.S. degree in electrical engineering from National Tsing Hua University, Hsinchu, Taiwan, in 2000, the M.S. degree in electronics engineering from National Chiao Tung University, Hsinchu, in 2002, and the Ph.D. degree in electrical engineering from the University of Southern California, Los Angeles, CA, USA, in 2008. From 2002 to 2003, he was with the Industrial Technology Research Institute, Hsinchu. In 2008, he was a Research Intern at the Mitsubishi Electric Research Laboratories, Cambridge, MA, USA. In 2009, he worked on NASA Small Business Innovation Research projects. Since 2010 he has been with the Research Center for Information Technology Innovation, Academia Sinica, Taipei, Taiwan, where he is currently an Assistant Research Fellow. He has published about 50 papers and has four granted U.S. patents. His research interests include wireless communications and networking. $\mathrm{He}$ was an Exemplary Reviewer for the IEEE Communications Letters in 2012. $\mathrm{He}$ has contributed to various conferences as a Technical Program Committee Member, including the IEEE International Conference on Communications 2012, 2013, and 2015. He was a recipient of the Best Paper Award from the IEEE Wireless Communications and Networking Conference 2012. 